Cahiers de philosophie de l'université de

\title{
Les catégories aristotéliciennes et la division de l'être : types de divisions et types d'ontologies
}

\section{Frédéric Nef}

\section{(2) OpenEdition}

12 Journals

Édition électronique

URL : https://journals.openedition.org/cpuc/1233

DOI : $10.4000 /$ cpuc. 1233

ISSN : 2677-6529

Éditeur

Presses universitaires de Caen

\section{Édition imprimée}

Date de publication : 31 décembre 2009

Pagination : 45-78

ISBN : 978-2-84133-340-0

ISSN : 1282-6545

\section{Référence électronique}

Frédéric Nef, «Les catégories aristotéliciennes et la division de l'être : types de divisions et types d'ontologies », Cahiers de philosophie de l'université de Caen [En ligne], 46 | 2009, mis en ligne le 03 septembre 2020, consulté le 31 janvier 2023. URL : http://journals.openedition.org/cpuc/1233 ; DOI : https://doi.org/10.4000/cpuc.1233

\section{(c) (†) 8}

Creative Commons - Attribution - Pas d'Utilisation Commerciale 4.0 International - CC BY-NC 4.0 https://creativecommons.org/licenses/by-nc/4.0/ 


\title{
Les catégories aristotéliciennes et la division de l'être: types de divisions et types d'ontologies
}

\author{
L'ontologie catégorielle a ses racines dans la doctrine aristotélicienne ${ }^{1}$ \\ exposée dans le traité éponyme et plus particulièrement dans le fameux \\ chapitre deuxième ${ }^{2}$. L'interprétation de ce texte bute notamment sur deux \\ problèmes: s'agit-il d'une division de la réalité ou de l'espace conceptuel?
}

1. L'origine est évidemment platonicienne (Philèbe 23c-27c): les genres de l'être sont la limite (peras), le mixte (mekton), la cause (aitia), l'illimité (apeiron). Les catégories aristotéliciennes ne sont pas des genres de l'être, ce qui a des conséquences pour la division: certains ont nié que les catégories divisent l'être, précisément parce qu'elles ne sont pas des genres de l'être et qu'elles ne communiquent pas, à la différence des genres de l'être platoniciens. Il est cependant courant de lire le contraire: «[...] l'étant est divisé en ce sens en dix catégories, l'essence doit être quelque chose de commun à toutes les natures, par lesquelles la multiplicité de l'étant est ordonnée à ses différents genres et espèces [...]» (saint Thomas d'Aquin, De Ente et Essentia, c. 1, Turin - Rome, Marietti, 1946, p. 9).

2. Je ne traiterai pas de la nature de la liste des dix catégories, simple énumération ou classement raisonné. Ammonios (Aristote, Les Attributions (catégories): le texte aristotélicien et les prolégomènes d'Ammonios d'Hermeias, Y. Pelletier (trad.), Montréal - Paris, Bellarmin - Les Belles Lettres, 1983, p. 91-92) soutient que la liste des dix catégories est un «dénombrement», tandis que la liste des quatre classes d'étants dans les Catégories (désormais Cat.), 2, serait une «division». Simplicius pense que la liste restreinte des quatre classes est un dénombrement: «La division en quatre termes n'est pas une division au sens propre, mais plutôt un dénombrement, comme si l'on désignait plusieurs hommes, en disant: "Ceux-ci sont des Lacédémoniens, ceux-là des Argiens" " (Simplicius, Commentaire sur les Catégories d'Aristote: chapitres II à IV, Ph. Hoffmann (trad.), Paris, Les Belles Lettres, 2001, p. 12). Cf. à la fin de l'article sur la division maximale et minimale (voir note 61). Boèce soutient que la liste des dix catégories est une enumeratio, car si c'était une division, les catégories diviseraient un genre unique, ce qui n'est pas le cas. Simplicius (ibid., p. 583-598) résume le problème et discute tous les sens de la division. J'ai discuté de la liste des catégories en relation avec la théorie brentanienne des catégories, contenue dans le recueil Kategorienlehre, dans «La lecture par Brentano des catégories aristotéliciennes et l'ontologie formelle », in Actes du colloque "Rôle et enjeux de la notion de catégorie en logique», D. Miéville (éd.), Neuchâtel, Centre de recherche sémiologique, Université de Neuchâtel (Travaux de Logique; 13), 1999, p. 63-92, travail complémentaire de celui-ci en ce qu'il indique ce que l'ontologie de Brentano a d'original tout en revendiquant sa fidélité à Aristote. 
Existe-t-il dans la doctrine d'Aristote des accidents individuels, des particuliers abstraits ${ }^{3}$ ? La réponse à ces questions détermine le sens de ce chapitre de l'histoire de l'ontologie. Je me propose de discuter de ces questions à la lumière de l'ontologie contemporaine. Les travaux récents de $\mathrm{R}$. Chisholm et de E. J. Lowe en l'ontologie catégorielle montrent l'actualité de la doctrine aristotélicienne: la réponse aux questions que nous venons de mentionner n'a pas seulement des conséquences historiques, mais peut contribuer à déterminer la place des catégories dans l'ontologie et plus largement à poser le problème du découpage catégoriel de la réalité. Je commencerai par examiner le sens de la division de l'être, puis je livrerai quelques réflexions sur le texte d'Aristote, avant d'exposer les développements des représentations semi-formelles sous forme de représentations géométriques (carrés, arbres, cubes), puis je discuterai des propositions de E. J. Lowe.

L'hypothèse que je serai conduit à évaluer consiste à soutenir que dans l'histoire de l'interprétation des Catégories il y a une corrélation, d'une part, entre le développement d'ontologies de constituant et les représentations sous forme d'arbres et, d'autre part, entre l'émergence d'ontologies des relations et les modèles sous forme de carrés. L'intérêt de ces carrés ontologiques est alors (si l'hypothèse a quelque vraisemblance) de contenir au moins en puissance une théorie des relations - une autre manière de voir les choses est de dire que ces représentations obligent à expliciter les relations entre les pôles des figures et jouent donc implicitement un rôle de catalyseur pour la réflexion.

\section{Qu'est-ce qu'un diviseur de l'être?}

Qu'est-ce que diviser? Qu'est-ce qu'une division? Diviser un nombre a (par exemple 45) par un nombre b (par exemple 3) c'est, par une procédure de calcul, déterminer combien de b il y a dans a. Le nombre divisé par le diviseur donne un quotient. Par exemple le nombre divisé 45 par le diviseur 3 donne le quotient 15 (ce qu'on affirme plus simplement en affirmant que 45 divisé par 3 donne 15 et que 15 est le résultat de la division). Si un nombre b est

3. Il est frappant de constater que les commentateurs néo-platoniciens ont consacré une partie non négligeable de leurs efforts à poser ces mêmes questions et à y répondre. On peut par exemple lire le commentaire par C. Luna de l'aporie de l'essence individuelle, p. 276-287 du commentaire de Simplicius (Simplicius, Commentaires sur les Catégories...). L'aporie des accidents séparables (ibid., p. 256-276) mériterait une comparaison avec des développements parallèles dans la métaphysique analytique. Pour commencer, un trait d'esprit de J.-F. Courtine: de Cambera, ou Sydney à... Athènes, Alexandrie et Byzance (cf. J.-F. Courtine, Inventio analogiae. Métaphysique et ontothéologie, Paris, Vrin, 2005, p. 8, n. 3: «De l'Ionie à Iéna disait l'autre. De Sydney, ou Cambera, à... ?»). 
une partie d'un nombre a, alors b peut mesurer a et on peut diviser a par b, puisque a est un multiple de $b$ (la division est l'opération inverse de la multiplication: si $\mathrm{a} \times \mathrm{b}=\mathrm{c}$, alors $\mathrm{c}: \mathrm{b}=\mathrm{a}$ et $\mathrm{c}: \mathrm{a}=\mathrm{b}$ ). Euclide ne déclare-t-il pas:

Un nombre est une partie d'un nombre, le plus petit du plus grand, lorsqu'il mesure le plus grand. [...] Un plus grand nombre est multiple d'un plus petit, lorsqu'il est mesuré par le plus petit ${ }^{4}$.

Le quotient peut être un nombre entier ou une fraction (par exemple: $22 / 7=3,142857 \ldots$ ). Dans l'arithmétique grecque la question des incommensurables est liée à la division: $\sqrt{2}$ en est l'exemple classique. Tout cela étant bien connu, il faut maintenant comparer diviser un nombre et diviser un étant.

L'expression «diviseur de l'être» est-elle une simple métaphore? Qu'estce que diviser l'être ou un être? Il ne faut pas confondre diviser et découper. Selon Platon (Phèdre, $\left.265^{\mathrm{e}}\right)$, le dialecticien découpe la masse articulée de la réalité comme un boucher une carcasse de bœuf, en suivant les articulations. On retrouve cette idée de découpage, suivant les articulations, dans le concept de propriétés naturelles de Lewis ${ }^{5}$. Ces dernières découpent la réalité «au bon endroit». Dans la métaphysique contemporaine, les propriétés naturelles qui découpent la réalité pourraient ainsi à bon droit être nommées des diviseurs de l'être.

La métaphore de la division se heurte à d'autres énigmes. Si nous divisons l'être, nous le divisons par quoi? Le divise-t-on par des diviseurs, ou au moyen des diviseurs? Que sont les diviseurs? Des parties de l'être ou des outils de découpage? Qu'est-ce en fin de compte qu'un diviseur? Le diviseur c'est le nombre de parties. Si la division est par exemple 750: 15, le diviseur est " 50 ", ce qui signifie qu'il y a 50 parties de 15 . Diviser c'est ainsi le contraire de rassembler, ranger les parties dans un tout: en ce sens diviser l'être c'est opposer ses déterminations, les jouer les unes contre les autres, ce qui s'oppose à une autre démarche, analogique ou harmonique qui vise à mettre en lumière des totalités.

4. Euclide, Les livres arithmétiques d'Euclide, Septième Élément, définitions 3 et 5, J. Itard (éd. et trad.), Paris, Hermann, 1961, p. 83.

5. Il s'agit de limiter le nombre des propriétés, de sélectionner parmi les propriétés rares, qui ne se multiplient pas de manière arbitraire, des propriétés qui jouent un rôle causal et explicatif. Les propriétés naturelles sont limitées en nombre par la pertinence des découpages qu'elles réalisent: ces découpages doivent correspondre aux articulations de l'explication scientifique (cf. D. Lewis, On the Plurality of Worlds, Oxford, Blackwell, 1986 p. 60 et F. Nef, Les propriétés des choses. Expérience et logique, Paris, Vrin, 2006, pour une discussion). En ce sens être composé de carbone est une propriété naturelle, alors qu'être situé à tel ou tel endroit n'est pas en général une propriété naturelle. 
Leibniz, dans la Correspondance avec Arnaud, répond à une objection d'Arnaud sur l'unité substantielle, portant sur le bris d'un carreau et pose à cette occasion la question de la division d'un être particulier en deux êtres:

Est-ce la forme substantielle d'un carreau de marbre qui fait qu'il est un? Si cela est, que devient cette forme substantielle quand il cesse d'être un, parce qu'on l'a cassé en deux? Elle est anéantie, ou elle est devenue deux ${ }^{6}$.

Leibniz répondra à une objection d'Arnaud, contre l'usage du concept de forme substantielle ${ }^{7}$, qu'un carreau de marbre ne jouit pas (tout comme les autres objets matériels) d'une véritable unité substantielle et donc que la division du carreau en deux ne pose pas réellement de problème: ce n'est pas une substance que l'on divise en deux, il s'agit d'un passage d'une unité par accident à une autre unité par accident (comme lorsqu'on divise un tas en deux). Il affirme donc:

[...] je crois qu'un carreau de marbre n'est peut-être que comme un tas de pierre, et ainsi ne saurait passer pour une seule substance, mais pour un assemblage de plusieurs. Car supposons qu'il y ait deux pierres, par exemple le diamant du Grand-Duc et celui du Grand-Mogol: on pourra mettre un nom même collectif ${ }^{8}$ en ligne de compte pour tous deux, et on pourra dire que c'est une paire de diamants, quoiqu'ils se trouvent bien éloignés l'un de l'autre; mais on dira pas que ces deux diamants composent une substance ${ }^{9}$.

Selon Leibniz, la division d'un étant n'est pas la même pour une unité accidentelle (unum per accidens) et substantielle. Une paire de diamants, un tas de pierre, mais aussi un carreau de marbre ou a fortiori une moitié d'un carreau de marbre sont des unum per accidens. Dans ce cas, la division

6. Leibniz, Discours de métaphysique et correspondance avec Arnauld, G. Le Roy (éd.), Paris, Vrin, 1966, p. 135.

7. L'objection cartésienne d'Arnaud visant la forme substantielle est la suivante: si l'unité d'un carreau de marbre est fonction de sa forme substantielle, que se passe-t-il lorsqu'un carreau est brisé en deux morceaux? Les deux réponses possibles sont également absurdes: la forme substantielle ne peut être détruite, si elle est substantielle et il est également absurde de supposer qu'on peut multiplier des formes substantielles par accident (lettre du 28 septembre 1686 , ibid., p. 135).

8. Un nom collectif désigne une collection (comme un «troupeau de moutons») et l'inférence de la sémantique à l'ontologie là aussi ne vaut pas: si x est désigné par un nom, cela ne signifie pas que $\mathrm{x}$ jouisse d'une unité ontologique ou substantielle. Le nom collectif complexe « une paire de diamants " peut désigner deux diamants réunis sous le terme de "paire», mais cette réunion sémantique sous un nom collectif ne fait pas que les deux diamants perdent leur individualité séparée et rentrent à titre d'ingrédients dans une substance complexe. Sur les noms collectifs dans la logique traditionnelle, voir par exemple la Logica Hamburgensis de Jungius (1657).

9. Lettre de Leibniz à Arnaud du 28 novembre-8 décembre 1686, ibid., p. 145. 
produit des unités accidentelles. Dans le cas des unités substantielles, la division peut soit détruire l'unité substantielle, car c'est la vie qui assure cette unité, soit séparer une partie accidentelle de la substance. Selon Leibniz, le carreau entier est une unité accidentelle et les deux moitiés de carreau, après la division, sont des unités accidentelles et non des moitiés de substance.

Quant à la division de l'étant dans sa totalité, un exemple est fourni par le Periphyseon (la Divisio Naturae) de Scot Érigène. Scot divise tous les êtres en quatre classes, suivant qu'un être est créateur ou pas, créé ou pas:

La division de la Nature selon quatre différences me semble comporter quatre espèces, dont la première consiste dans la Nature qui crée et qui n'est pas créée, la deuxième dans la Nature qui est créée et qui crée, la troisième dans la Nature qui est créée et qui ne crée pas, la quatrième dans la Nature qui ne crée pas et qui n'est pas créée ${ }^{10}$.

\begin{tabular}{|c|c|c|}
\hline Créateur & & Créé \\
\hline+ & Dieu créateur & - \\
\hline+ & Causes premières & + \\
\hline- & Nature & + \\
\hline- & $\begin{array}{c}\text { Dieu comme terme } \\
\text { du mouvement }\end{array}$ & - \\
\hline
\end{tabular}

La division érigénienne repose donc sur le concept de création et elle divise jusqu'à Dieu, en créateur et non créateur. La natura érigénienne comprend absolument tout, sa division est donc une division totale de l'étant. Mais dans ce cas que sont les diviseurs de l'être? Le créateur et le créé, ou les quatre classes d'êtres?

Kant dans une note de l'introduction à la Critique de la faculté de juger livre une réflexion sur son usage de la division (Einteilung) en répondant au reproche d'utiliser des trichotomies, probablement parce que la logique traditionnelle depuis Platon était fondée sur des dichotomies. Selon lui une division peut être bipartite ou tripartite (il ne considère pas la quadripartition). Alors que l'empirisme ou l'idéalisme aboutissent à des bipartitions génératrices d'antinomies, l'idéalisme transcendantal use nécessairement de tripartitions ${ }^{11}$ dont le modèle est: condition, conditionné, union de la condition et du conditionné:

10. Jean Scot Erigène, De la division de la nature, F. Bertin (trad.), Paris, PUF, 1995, vol. 1, p. 66.

11. La dialectique hégélienne et la sémiotique peircienne ont toutes deux exploité cette voie kantienne de la tripartition (la première période de la philosophie de C. S. Peirce étant par exemple fondée sur la triade Moi-Nature-Dieu). 
On a émis des doutes sur le fait que mes divisions soient presque toujours tripartites en philosophie pure. Mais cela réside dans la nature de la chose. Si une division doit se faire a priori ou bien elle sera analytique, selon le principe de contradiction, et alors elle sera toujours bipartite (quodlibet ens aut $A$ aut non A) ou bien elle sera synthétique; et si dans ce cas elle doit être conduite à partir de concepts a priori [...] il faut selon, ce qui est en général requis en vue de l'unité synthétique en général, à savoir $1^{\circ}$ la condition, $2^{\circ}$ un conditionné, $3^{\circ}$ le concept qui résulte de l'union du conditionné avec sa condition, que la division soit nécessairement une dichotomie ${ }^{12}$.

Pour Kant donc, il n'y a pas une bonne (ternaire) et une mauvaise (binaire) division: il faut distinguer deux types de divisions selon le type de jugement, analytique ou synthétique.

Les universaux, les genres de l'être et les transcendantaux découpent certainement le champ conceptuel, lexical de l'ontologie, mais peut-on affirmer en toute rigueur qu'ils divisent l'être? On comprend ce qu'est diviser (enteilen) un étant: transformer une unité (Ein) en parties (Teilen); si on prend la métaphore de la division au sérieux, l'être est l'arrière-fond du découpage des étants: en un certain sens, ce sont les étants qui découpent l'être - les étants et non les concepts sont les véritables diviseurs de l'être. Il faudrait alors distinguer une division de l'être par les étants et un découpage subséquent des étants au moyen des universaux, transcendantaux, etc.

On réfléchira ici sur l'idée de division elle-même et on prendra comme exemple la division la plus complexe, la quadripartition, car l'hypothèse que je discuterai c'est que la division ontologique qui est nécessairement une division à quatre termes et qui est le produit d'une double division entre universel et particulier, abstrait et concret contient en germe une doctrine des structures ontologiques, composées d'entités élémentaires et de relations (dépendance, séparation, individualisation...).

\section{Ramification $v s$ carréification et constituants $v s$ relations}

J'introduirai maintenant deux distinctions conceptuelles importantes. La première est entre ramification et carréification. Une ramification consiste à opérer une suite finie de dédoublements et de divisions, à mettre en place un graphe conceptuel. Par exemple:

12. E. Kant, Critique de la faculté de juger, introduction, IX, note 2, Euvres philosophiques, Paris, Gallimard (La Pléiade), 1985, t. II, p. 955. 


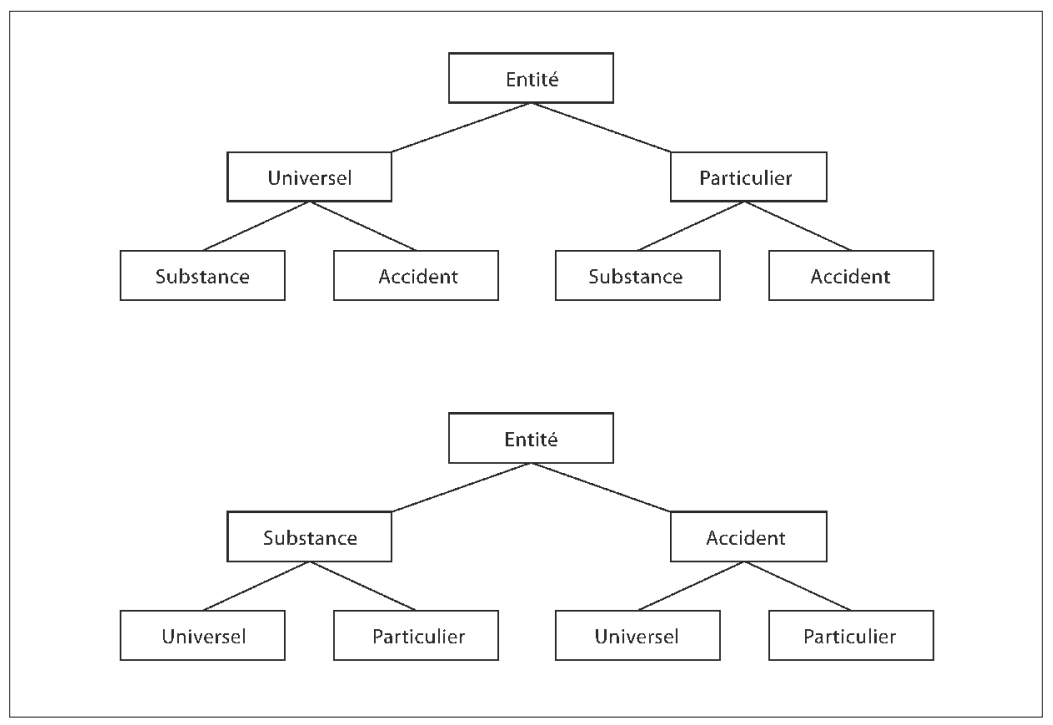

La division ontologique se trouve à son origine chez Platon (notamment dans les passages fameux du Politique, du Philèbe et du Sophiste) et prend une forme explicitement ramifiée dans l'«arbre de Porphyre» (Isagoge ${ }^{13}$ ) qui de division en division passe de l'être à l'individu. On distingue division (diaresis) avec reste (blanc et noir) et division sans reste (blanc et non blanc): on peut distinguer les contraires et les contradictoires par l'existence ou pas d'un reste de la division.

La carréification ${ }^{14}$ consiste à présenter sous la forme d'une quadripartition un domaine de l'être, voire tout le domaine de l'être. On voit apparaitre le carré ontologique sous forme de figure (paradeigma) schématisant les divisions, oppositions et relations de Catégories, 2, chez Ammonios ${ }^{15}$, puis Philopon, et bien sûr Boèce. À une ramification correspond une carréification: par exemple la deuxième des deux ramifications immédiatement ci-dessus peut être carréifiée comme suit:

13. Cf. Porphyre, Introduction, J. Barnes (trad., introd. et comment.), Oxford, Clarendon Press, 2003.

14. On pourrait préférer à ce terme barbare le terme de «quadrature» (cf. «la quadrature du cercle » et non la "carréification du cercle»), mais le terme de "quadrature » est attaché à un problème géométrique précis et désigne non une division mais une inscription (découvrir une procédure calculatoire pour inscrire un cercle dans un carré).

15. On trouve le schéma d'Ammonios (Aristote, Les Attributions..., p. 92) commenté dans Simplicius, Commentaire sur les Catégories..., p. 162. 


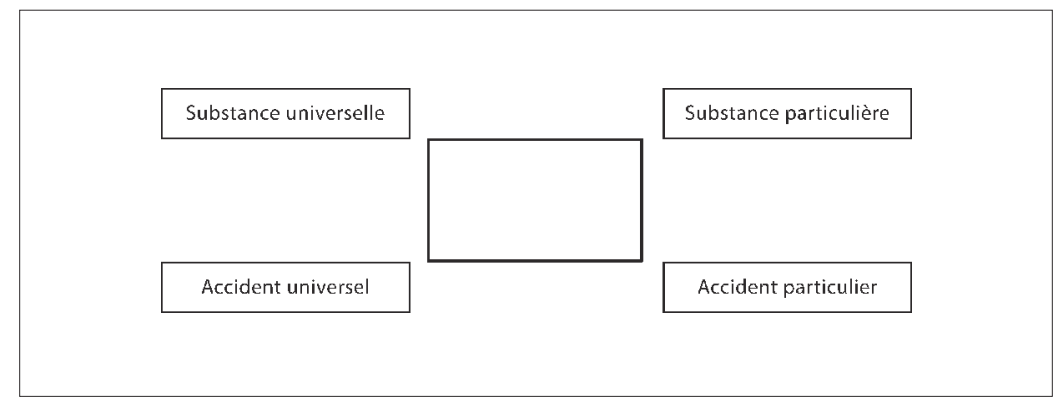

La deuxième distinction conceptuelle que j'introduis est celle entre ontologie de constituant et ontologie de relation ${ }^{16}$. Une ontologie de constituant, comme celle d'Aristote est une ontologie qui vise à mettre à jour les structures ontologiques fondamentales constituant la réalité, notamment tout ce qui a trait à la division fondamentale en substances et accidents. Dans une telle ontologie ce qui compte est la spécification progressive de l'être. Dans une ontologie de relations au contraire, les entités ne sont pas des points de départ spécifiés par les divisions ultérieures, comme par exemple dans l'arbre de Porphyre, mais des nœuds simplement de relations. Par exemple, chez Platon les particuliers concrets, les choses sensibles tiennent leur être (ou leur semblant d'être, leur paraitre, l'apparaître de leur forme) de leur participation à des Formes, ou Idées et dans cette ontologie de relation on peut soutenir que les choses sensibles ne sont que des nœuds de relations (ici la somme des relations de participation à une multiplicité de Formes prédiquées).

J'avance l'hypothèse que la ramification est un mode de représentation ou de division privilégié des ontologies du constituant, tandis que la carréification a des affinités avec les ontologies de la relation ${ }^{17}$. La première raison qui vient à l'esprit est la suivante: dans une ramification, la division qui dérive une paire d'opposés d'un nœud hiérarchiquement supérieur est répétée de niveaux en niveaux descendants et il n'y a pas de spécification des relations dans ce genre de taxinomie. Dans un carré ontologique, les sommets du carré sont liés par des relations qui sont différentes selon que l'on considère les sommets pris deux à deux comme horizontaux, verticaux

16. M. Loux, "Aristotle's Constituent Ontology», in Oxford Studies in Metaphysics, D. Zimmermann (éd.), Oxford, Clarendon Press, 2006, vol. 2, p. 207-250.

17. Historiquement cela permettrait peut-être d'éclairer les deux modes de représentations de divisions, le premier de Boèce (carré), le second de Porphyre (arbre), chez deux auteurs marqués par le platonisme et s'attachant à donner une cohérence à l'héritage aristotélicien (catégories) et platonicien (dialectique). 
ou sur une diagonale. L'hypothèse que l'on pourrait émettre est que la carréification n'est pas une démarche formaliste et stérile, mais une étape d'une démarche plus large qui s'efforce de déterminer les relations entre les éléments de base de l'ontologie (atoma, eskata, kategorema) ${ }^{18}$.

J'examinerai les enjeux de la division de l'être en ce qui concerne les catégories aristotéliciennes, à la fois chez Aristote même, certains commentateurs et dans la littérature métaphysique contemporaine. Je montrerai ce qu'il en est de la carréification, dans son rapport à la ramification. Je tenterai de déterminer si la division de l'être est plus qu'une métaphore, si elle peut servir à dévoiler des structures ontologiques.

\title{
Commentaire de Cat. 1a 20 sq.: les catégories, divisions de l'être ou découpage de l'espace conceptuel?
}

\author{
Voici le texte d'Aristote, dans les traductions de J. Tricot ${ }^{19}$, R. Bodéüs ${ }^{20}$ \\ et de Y. Pelletier ${ }^{21}$; je fais suivre ces traductions de la version latine de \\ Boèce:
}

18. L'atoma est le terme d'une division. Dans les Topiques, Aristote met en place la division par des prédicats des groupes d'individus, jusqu'à arriver à quelque chose de non divisible (qu'on désigne par le terme de "espèce infime»): «Un autre lieu, c'est d'examiner tous les cas où un prédicat a été affirmé ou nié universellement d'un sujet. Mais l'examen de ces cas doit porter sur les espèces et non sur la multitude infinie des individus, car alors l'enquête se fera d'une façon plus méthodique et par des étapes moins nombreuses. Et il faut procéder à cet examen en commençant par les groupes primordiaux et descendre progressivement jusqu'aux espèces indivisibles" (Aristote, Topiques, II, 2, 109 b 13-18, J. Tricot (trad.), Paris, Vrin, 1965, p. 55-56.). Dans le commentaire de Simplicius (Commentaires sur les Catégories..., p. 294) on trouve une précision importante: l'atoma peut être soit un accident, soit une essence. L'eskaton est lui le particulier, par exemple dans le syllogisme pratique du médecin (Aristote, Métaphysique, Z, 1035 b 30). Cf. Aristote, Éthique à Nicomaque, VI, vii, 7 et III, iii, 12. On trouve en 1147 a 3 une formulation un peu différente: "prakta gar ta kath'eskata?» («les objets de l'action ne sont-ils pas l'individuel?»). Une caractéristique de la phronesis est qu'elle n'a pas pour objet les universels mais elle doit avoir la connaissance des faits particuliers, car elle est de l'ordre de l'action et l'action a rapport aux choses singulières (praxis peri kath eskata). Le terme eskata se rencontre par exemple dans le texte célèbre de la Métaphysique, Z, sur l'art du médecin mentionné supra, quand le médecin, dans son raisonnement, remonte aux «éléments ultimes et derniers». L'eskaton est le singulier, au sens de terme dernier d'une série. Dans une action qui est le fruit d'une délibération il y a d'une part les principes de l'action qui sont généraux (par exemple: il faut conserver l'équilibre pour la santé) et d'autre part les choses singulières, personnes, temps, lieu, circonstances (telle constitution, telle saison pour reprendre un exemple médical).

19. Aristote, Organon, I. Catégories, II. De l'interprétation, J. Tricot (trad.), Paris, Vrin, 1959.

20. Aristote, Catégories, avec le texte grec, R. Bodéüs (trad. et introd.), Paris, Les Belles Lettres, 2001.

21. Aristote, Les Attributions... 


\begin{tabular}{|c|c|c|}
\hline Traduction Tricot & Traduction Bodéüs & Traduction Pelletier \\
\hline $\begin{array}{l}\text { Parmi les êtres, les uns } \\
\text { sont affirmés d'un sujet, } \\
\text { tout en n'étant dans } \\
\text { aucun sujet: }\end{array}$ & $\begin{array}{l}\text { D'entre les êtres, les uns } \\
\text { se disent d'un certain } \\
\text { sujet, mais ne sont } \\
\text { inhérents à aucun sujet. }\end{array}$ & $\begin{array}{l}\text { Parmi les êtres, certains } \\
\text { se disent d'un sujet, } \\
\text { mais ne sont en } \\
\text { aucun sujet; }\end{array}$ \\
\hline $\begin{array}{l}\text { par exemple homme est } \\
\text { affirmé d'un sujet, savoir } \\
\text { d'un certain homme, } \\
\text { mais il n'est dans } \\
\text { aucun sujet. }\end{array}$ & $\begin{array}{l}\text { Ainsi l'homme se dit } \\
\text { bien d'un sujet, un } \\
\text { certain homme, mais } \\
\text { il n'est inhérent } \\
\text { à aucun sujet. }\end{array}$ & $\begin{array}{l}\text { par exemple l'homme } \\
\text { se dit d'un sujet, tel } \\
\text { homme, mais n'est } \\
\text { en aucun sujet. }\end{array}$ \\
\hline $\begin{array}{l}\text { D'autres sont dans } \\
\text { un sujet, mais ne sont } \\
\text { affirmés d'aucun sujet }\end{array}$ & $\begin{array}{l}\text { D'autres en revanche } \\
\text { sont inhérents à un } \\
\text { sujet, mais ne se disent } \\
\text { d'aucun sujet }\end{array}$ & $\begin{array}{l}\text { D'autres sont en un } \\
\text { sujet, mais ne se disent } \\
\text { d'aucun sujet. }\end{array}$ \\
\hline $\begin{array}{l}\text { (par dans un sujet } \\
\text { j'entends ce qui ne se } \\
\text { trouvant pas dans un } \\
\text { sujet comme sa partie, } \\
\text { ne peut être séparé de } \\
\text { ce en quoi il est): }\end{array}$ & $\begin{array}{l}\text { (par «inhérent à un } \\
\text { sujet» je veux dire ce } \\
\text { qui, sans se trouver en } \\
\text { quelque sorte à titre de } \\
\text { partie, ne peut exister } \\
\text { à part de la chose } \\
\text { où il est); }\end{array}$ & $\begin{array}{l}\text { Par en un sujet } \\
\text { j'entends ce qui étant } \\
\text { en quelque chose sans } \\
\text { que ce soit à titre de } \\
\text { partie ne peut être } \\
\text { séparément de ce } \\
\text { en quoi il est. }\end{array}$ \\
\hline $\begin{array}{l}\text { par exemple une } \\
\text { certaine science } \\
\text { grammaticale existe } \\
\text { dans un sujet, savoir } \\
\text { dans l'âme, mais elle } \\
\text { n'est affirmée d'aucun } \\
\text { sujet; et une certaine } \\
\text { blancheur existe dans } \\
\text { un sujet, savoir } \\
\text { dans le corps }\end{array}$ & $\begin{array}{l}\text { ainsi une certaine } \\
\text { science des lettres est } \\
\text { bien inhérente au sujet, } \\
\text { l'âme, mais elle se dit } \\
\text { d'aucun sujet et une } \\
\text { certaine blancheur est } \\
\text { bien inhérente à } \\
\text { un sujet, le corps, }\end{array}$ & $\begin{array}{l}\text { Par exemple, telle } \\
\text { science grammaticale } \\
\text { est en un sujet, l'âme, } \\
\text { mais ne se dit d'aucun } \\
\text { sujet; et tel blanc est } \\
\text { en un sujet, le corps }\end{array}$ \\
\hline $\begin{array}{l}\text { (car toute couleur } \\
\text { est dans un corps); } \\
\text { et pourtant elle n'est } \\
\text { affirmée d'aucun sujet. }\end{array}$ & $\begin{array}{l}\text { puisque toute couleur } \\
\text { est inhérente au corps, } \\
\text { mais elle ne se dit } \\
\text { d'aucun sujet. }\end{array}$ & $\begin{array}{l}\text { - de fait toute couleur } \\
\text { est en un corps - mais } \\
\text { ne se dit d'aucun sujet. }\end{array}$ \\
\hline $\begin{array}{l}\text { D'autres êtres sont à la } \\
\text { fois affirmés d'un sujet } \\
\text { et dans un sujet: }\end{array}$ & $\begin{array}{l}\text { D'autres à la fois se } \\
\text { disent d'un sujet et sont } \\
\text { inhérents à un sujet. }\end{array}$ & $\begin{array}{l}\text { D'autres se disent d'un } \\
\text { sujet et sont dans } \\
\text { un sujet; }\end{array}$ \\
\hline
\end{tabular}




\begin{tabular}{|c|c|c|}
\hline Traduction Tricot & Traduction Bodéüs & Traduction Pelletier \\
\hline $\begin{array}{l}\text { par exemple la Science } \\
\text { est dans un sujet, savoir } \\
\text { dans l'âme et elle est } \\
\text { aussi affirmée d'un } \\
\text { sujet, la grammaire. }\end{array}$ & $\begin{array}{l}\text { Ainsi la science est } \\
\text { inhérente à un sujet, } \\
\text { l'âme, et elle se dit d'un } \\
\text { sujet, la connaissance } \\
\text { des lettres. }\end{array}$ & $\begin{array}{l}\text { par exemple la science } \\
\text { est en un sujet, l'âme, } \\
\text { et se dit d'un sujet, } \\
\text { la grammaire. }\end{array}$ \\
\hline $\begin{array}{l}\text { D’autres êtres enfin, ne } \\
\text { sont ni dans un sujet, } \\
\text { ni affirmés d'un sujet, }\end{array}$ & $\begin{array}{l}\text { D'autres enfin ne sont } \\
\text { ni inhérents à un sujet, } \\
\text { ni dits d'un sujet. }\end{array}$ & $\begin{array}{l}\text { D'autres enfin ne sont } \\
\text { pas en un sujet et ne se } \\
\text { disent pas d'un sujet; }\end{array}$ \\
\hline $\begin{array}{l}\text { par exemple cet homme, } \\
\text { ce cheval, }\end{array}$ & $\begin{array}{l}\text { Ainsi un certain } \\
\text { homme, ou un } \\
\text { certain cheval. }\end{array}$ & $\begin{array}{l}\text { par exemple tel homme } \\
\text { ou tel cheval. }\end{array}$ \\
\hline $\begin{array}{l}\text { car aucun être de cette } \\
\text { nature n'est dans un } \\
\text { sujet, ni affirmé } \\
\text { d'un sujet }\end{array}$ & $\begin{array}{l}\text { Aucun des êtres de ce } \\
\text { genre, en effet, n'est } \\
\text { inhérent à un sujet, ni } \\
\text { ne se dit d'un sujet. }\end{array}$ & $\begin{array}{l}\text { Car aucun être de cette } \\
\text { sorte n'est en un sujet, } \\
\text { ni se dit d'un sujet. }\end{array}$ \\
\hline $\begin{array}{l}\text { - Et absolument } \\
\text { parlant, les individus } \\
\text { et ce qui est numéri- } \\
\text { quement un ne sont } \\
\text { jamais affirmés } \\
\text { d'un sujet; }\end{array}$ & $\begin{array}{l}\text { Dans l'absolu } \\
\text { d'ailleurs, les êtres } \\
\text { individuels et } \\
\text { numériquement } \\
\text { uns ne se disent } \\
\text { d'aucun sujet, }\end{array}$ & $\begin{array}{l}\text { Enfin, c'est de façon } \\
\text { absolue que les êtres } \\
\text { individuels et } \\
\text { numériquement } \\
\text { uns ne se disent } \\
\text { d'aucun sujet, }\end{array}$ \\
\hline $\begin{array}{l}\text { pour certains toutefois } \\
\text { rien n'empêche qu'ils } \\
\text { ne soient dans } \\
\text { un sujet, }\end{array}$ & $\begin{array}{l}\text { mais rien n'empêche } \\
\text { quelques-uns d'être } \\
\text { inhérents à } \\
\text { un sujet. }\end{array}$ & $\begin{array}{l}\text { mais rien n'empêche } \\
\text { quelques-uns d'entre } \\
\text { eux d'être en } \\
\text { un sujet; }\end{array}$ \\
\hline $\begin{array}{l}\text { car une certaine science } \\
\text { grammaticale est dans } \\
\text { un sujet (mais n'est } \\
\text { affirmée d'aucun sujet). }\end{array}$ & $\begin{array}{l}\text { Une certaine } \\
\text { science des lettres, en } \\
\text { effet, compte parmi les } \\
\text { réalités inhérentes } \\
\text { à un sujet. }\end{array}$ & $\begin{array}{l}\text { en effet telle science } \\
\text { grammaticale } \\
\text { appartient aux } \\
\text { êtres qui sont } \\
\text { en un sujet }\end{array}$ \\
\hline (nouv. éd., 1969, p. 4) & (2001, p. 4) & $(1983$, p. 24$)$ \\
\hline
\end{tabular}


Traduction de Boèce:

Eorum quae sunt alia de subjecto quodam dicuntur, in subjecto vero nullo sunt, ut homo de subjecto quidem dicitur aliquo homine, in subjecto vero nullo est; alia autem in subjecto quidem sunt, de subjecto vero nullo dicuntur (in subjecto autem esse sine eo in quo est), ut quaedam grammatica in subjecto quidem est in anima, de subjecto vero nullo dicitur, et quodam album in subjecto est in corpore (ominis etim color in corpore est); alia vero et de subjecto dicuntur et in subjecto sunt, ut scientia in subjecto quidem est in anima, de subjecto vero dicitur de grammatica; alia vero neque in subjecto sunt neque de subjecto dicuntur, ut aliquis homo vel aliquis equus; nihil enim horum neque in subjecto est neque de subjecto dicitur. Simpliciter autem quae sunt individua et numero singularia nullo de subjecto dicuntur, in subjecto autem nihil ea prohibet esse; quaedam enim grammatica in subjecto est ${ }^{22}$.

La question préliminaire est: de quoi parle ce texte? Simplicius dans son Commentaire sur les Catégories a dressé un panorama des différentes réponses à cette question. On sait que certains ont répondu: les étants; d'autres: les concepts; d'autres, enfin: les modes de prédication et d'attribution (ces derniers donnant une portée principalement sémantique au texte). Simplicius ${ }^{23}$ résume ainsi les positions de certains commentateurs sur la portée (skopos) du texte des Catégories:

- Jamblique $\rightarrow$ pragmata,

- Philopon et David (Elias) $\rightarrow$ phonai,

- Olympiodore $\rightarrow$ noemata ${ }^{24}$.

Simplicius est partisan de l'interprétation dite du «triple skopos» : ce texte porterait à la fois sur les choses, les mots et les concepts.

À l'époque actuelle, R. Bodéüs et A. de Libéra donnent par exemple des réponses différentes et opposées et, reproduisant cette gamme d'interprétations, en montrent la pérennité. Il faut, avant de rappeler leur instructive divergence, rappeler que l'interprétation grammaticale avait fini par prédominer avant l'interprétation d'Ackrill qui rompait avec une longue tradition d'absence de commentaires à l'époque moderne.

22. Aristote, Aristoteles latinus, vol. 1, Categoriae vel Praedicamenta, L. Minio-Paluello (éd.), Bruges - Paris, Desclée de Brouwer, 1961, p. 5-6.

23. Simplicius, Commentaire sur les Catégories..., p. 80.

24. Il est frappant de constater que l'on retrouve en germe la distinction médiévale des nominalistes (Philopon/Occam), des réalistes (Jamblique/Duns Scot) et des conceptualistes (Olympiodore / Abélard), en ce qui concerne les universaux, ce qui est normal si les universaux et les catégories appartiennent à la même sphère. Les travaux de A. de Libera ont fourni de quoi documenter la connexion entre ces deux classements. 
Pour R. Bodéüs,

les «catégories» sont simplement les différents genres d'indications fournies par les imputations ${ }^{25}$ [kategoremata] que supposent l'attribution d'un sujet de son espèce ou de son genre ${ }^{26}$.

Aristote parle cependant de kategoriai ton ontos dans un texte important:

Nous avons traité de l'Être pris au sens premier et auquel se rapporte toutes les autres catégories de l'Être; autrement dit nous avons traité de la substance ${ }^{27}$.

C'est à travers une comparaison détaillée avec les Topiques (spécialement I, 9, la détermination des «catégories des prédications»- ta géné ton kategorion ${ }^{28}$-) que R. Bodéüs est conduit à rapprocher ce texte des Catégories et à conclure que ce texte est une «topique définitionnelle», ce qui expliquerait son début abrupt, consistant en une suite de définitions de relations sémantiques ${ }^{29}$. Ce qui précède immédiatement notre texte est une reprise de la distinction platonicienne entre expressions ou combinaisons avec liaison ou sans liaison. Le fait qu'Aristote continue par cette quadripartition peut s'interpréter ainsi : il veut montrer que même si cette distinction issue du Théétète mérite d'être reprise, il est nécessaire de la développer. La sémantique platonicienne repose sur un entrelacement du nom et du verbe, entrelacement pensé comme une composition méréologique. Aristote passe d'une sémantique à deux termes à une sémantique à trois termes, le lien

25. R. Bodéüs traduit kategoria par imputation (cf. Cat., 3 a 35) probablement pour ne pas s'engager dans la lignée des interprétations.

26. Aristote, Catégories, R. Bodéüs (trad. et introd.), introduction, p. LXXXV.

27. Aristote, Métaphysique, $\Theta, 1,1045$ b 28, J. Tricot (trad.), Paris, Vrin, 1974, t. 2, p. 482-483.

28. Aristote, Topiques, J. Tricot (trad.), p. 20. On peut remarquer que, dans ce texte, les catégories des prédications expriment des essences. Aristote affirme qu'utiliser une catégorie $\mathrm{K}$ à propos d'une essence $\mathrm{E}$ d'une chose $\mathrm{C}$ c'est exprimer $\mathrm{E}$ et désigner $\mathrm{C}$.

29. Cela confirme aussi la thèse radicale de ceux, peu nombreux, qui voient dans ce texte un compendium scolaire, voire un texte non aristotélicien. Je me range du côté de ceux qui avouent ne pas reconnaître le style de la Métaphysique dans cet opuscule: Aristote ne dit pas quels sont les antécédents de ses distinctions, ne cherche même pas à les asseoir sur des définitions et il ne les soumet pas à l'épreuve ensuite, toutes les étapes habituelles de sa démarche: il n'y a pas de dimension jurisprudentielle ou aporétique. Cela dit, même si ce texte est un simple recueil de définitions à usage scolaire, même si la quadripartition du deuxième chapitre est une simple topique, la question se pose de son interprétation, au vu de son intégration dans l'Organon, et plus largement dans le corpus aristotélicien (puis chez les commentateurs). Lowe récemment (The Four-Category Ontology, Oxford, Oxford University Press, 2006) a vu dans le chapitre 2 des Catégories "peut-être le texte le plus important de l'histoire de l'ontologie» (p.58). En termes quantitatifs certainement: probablement aucun texte, à part peut-être le passage de l'Isagoge sur les universaux, n'a déchaîné une telle fureur exégétique. 
entre sujet et attribut étant assumé par le verbe être, qui se dit de multiples façons - nous voici alors au fondement du déploiement catégoriel.

Le texte opère une double division:

(i) (l') homme vs une certaine blancheur, une certaine science des lettres,

(ii) la science ${ }^{30} v s$ un certain homme, un certain cheval.

Chacune de ces divisions oppose un universel et un particulier. L'opérateur de particularité est tinos («un certain», ou «tel» suivant les traductions).

Simplicius pose la question de la nature de la division dans ce texte, dans son Commentaire sur les Catégories:

Mais comment qualifier ce découpage (tomé) qui a abouti aux genres génériques? est-ce une division (diaresis) ? Et comment serait-ce une division, s'il ne s'agit ni de diviser un genre en espèces [comme dans un arbre de Porphyre], ni de diviser un tout en parties? [...] Est-ce donc que cette division ressemble à celle des troupes qui dans l'armée sont rangées par compagnies, si bien que comme le capitaine commande la compagnie, l'essence commande les essences et chacune des autres catégories la multiplicité qui lui est subordonnée?

Il est remarquable qu'Aristote ne donne pas de noms pour ces quatre types ou classes d'êtres ou d'étants, ces genres génériques pour reprendre l'expression ci-dessus ${ }^{31}$. L'exception est celle des êtres individuels (ta atoma, 1 b 6). Toutefois on peut penser que «individuels» n'est qu'une caractéristique de cette classe d'êtres, sans que «êtres individuels» désigne clairement cette classe. Ta atoma veut simplement dire qu'on ne peut aller plus loin dans la descente des genres aux espèces et des espèces aux individus.

Y a-t-il dans ce texte une optique particulariste - en entendant par "particularisme» la doctrine ontologique qui admet l'existence de particuliers comme entités de base? Le fait que la substance première (proté ousia) soit nommée individu (atomon) semble aller dans ce sens. Mais on parle de particularisme quand il y une doctrine non seulement de l'individualité de la substance, mais aussi de l'individualité de l'accident («non-substance», comme le dit, bizarrement, R. Bodéüs), c'est-à-dire

30. Ou «la connaissance» (trad. Lallot et Ildefonse).

31. Les commentateurs grecs se sont posés la question et se sont divisés sur la réponse à donner: Porphyre pense qu'Aristote a voulu utiliser des locutions simples (être dans, être dit de) plutôt que d'utiliser des termes techniques (essence, particulier...) alors que les Alexandrins pensent qu'au contraire Aristote a cultivé l'obscurité avec ces locutions non définies. Cf. Simplicius, Commentaire sur les Catégories, p. 140 sq. 
quand il y a particularisation ontologique (et pas simplement sémantique) des attributs et des propriétés. Ce qui est en cause est donc une certaine blancheur ou une certaine science des lettres (Aristote prend soin de choisir deux exemples hétérogènes, l'un physique, l'autre psychique). Le texte porte tis grammatiké et ti leukon que l'on peut aussi traduire «telle science des lettres» et «telle blancheur» (ou «telle pâleur», cf. les trois traductions supra). Le démonstratif est vraiment un opérateur de particularité et Ackrill a soutenu que tis grammatiké et ti leukon sont d'authentiques particuliers ontologiques d'où le nom de «propriétés ackrilliennes » pour désigner ces propriétés aristotéliciennes et individuelles:

Aristotle could not say that generosity is in Callias as subject, since there could be generosity without any Callias. Only this individual generosity - Callia's generosity - is in Callias. Equally, white is not in chalk as subject, since there could be white even if there were no chalk. White is in body, because every individual white is the white of some individual body ${ }^{32}$.

J'ai soutenu ${ }^{33}$ que cette interprétation posait des problèmes et j'ai opposé un particularisme platonicien authentique et un universalisme aristotélicien. Bien sûr, Aristote admet dans son ontologie des particuliers concrets, c'està-dire des substances individuelles composées de matière et de forme, de puissance et d'acte, mais c'est, on le sait, une difficulté pour lui de savoir sur quoi est fondée la particularité de ces substances, ce qu'on appelle la question de l'individuation par la forme ou la matière (cf. les travaux de J. Gracia). De plus, on le sait, il existe une contradiction entre l'universalisme épistémologique (il n'y a de science que de l'universel et du nécessaire) et le particularisme épistémologique. Cette contradiction est d'autant plus gênante, et on a d'autant plus le désir de la résorber que le réalisme aristotélicien affirme, au moins implicitement, la primauté de l'ontologique sur l'épistémique, ou l'épistémologique. Les principes ultimes sont autant ontologiques que logiques, l'idéal théorétique de la philosophie première engage à une ontologie du premier moteur, entre mouvement et désir, comme on peut le voir en Métaphysique, $\Lambda, 9$. Si c'est le cas, si effectivement Aristote n'admet pas une classe ontologique de propriétés particulières, on peut se demander si le texte déploie une ontologie et pas seulement une topique catégorielle ou définitionnelle.

32. Aristote, "Categories» and «De Interpretatione», J. L. Ackrill (trad. et comment.), Oxford, Oxford University Press, 1963, p. 74.

33. Dans «Platonisme et particularisme: à propos d'une histoire des propriétés individuelles ou pourquoi Aristote a tort et Platon a raison ", Cahiers de Philosophie de l'Université de Caen, vol. 38-39, 2003, S. Chauvier et V. Carraud (dir.), p. 159-186. 
On peut aussi remarquer qu'Aristote lui-même ne dit rien sur les relations entre les pôles de sa combinatoire (ce qui, incidemment, renforce l'aspect «topique») - ce sont les commentateurs qui calqueront le carré logique sur le carré ontologique (voir infra). En cela la quadripartition aristotélicienne est assez étrange: comme toute carréification elle contient implicitement une ontologie relationnelle, mais considérée plus strictement, en tant que structure ontologique que l'on peut explicitement dégager du texte des Catégories, elle est apparemment dépourvue de relations. On pourrait rétorquer que les relations «être dans $(\mathrm{x}, \mathrm{y})$ » $v s$ «être dit de $(\mathrm{x}, \mathrm{y})$ » sont d'authentiques relations ontologiques et donc qu'il y a bien ici une ontologie relationnelle. Cependant ce sont plus des relations formelles qui servent à établir des critères, comme dans une topique, que des relations au sens plein qui constitueraient les entités comme connexions de relations ${ }^{34}$.

La relation d'inhérence est donc caractérisée à la fois négativement et positivement. Négativement: la relation d'inhérence est différente de celle de partie/tout ${ }^{35}$. Cela signifie par exemple que si la science des lettres est dans l'âme (ou la blancheur dans le corps) cette science n'est pas une partie du corps. En ce qui concerne la science des lettres, cela pose le problème de la nature de la disposition exprimée par cette propriété: si cette science est dans l'âme, c'est forcément à titre de disposition, or il semble curieux à première vue qu'une disposition soit un universel concret. Quelle est la relation exacte entre l'âme et sa science?

Positivement, cette relation d'inhérence est caractérisée comme une relation de dépendance. Aristote distingue «exister à part» (koris einai) qui est le propre de la substance (seule la substance est indépendante) d' "exister dans» qui est le propre des non-substances. Être inhérent, c'est être dépendant au sens de non séparé. Mais comme le remarque $\mathrm{R}$. Bodéüs ${ }^{36}$ cette détermination est également celle des parties de la substance, car la partie est dans le tout et ne peut exister sans le tout sinon de manière équivoque (cf. Politique, I, 2, 1253 a 20-22). Or Aristote stipule que les parties du corps sont dites des substances (Métaphysique, $\Delta, 8,1017$ b 12; Z, 2, 1028 b 9; H, 1, 1042 a 10). C'est pour cette raison qu'Aristote a commencé par donner la condition négative.

34. G. Bergmann a développé une ontologie de la connexion à travers son concept de nexus, une ontologie du primat de la relation. Cf. G. Bergmann, New Foundations of Ontology, W. Heald (éd.), Madison, Wisconsin University Press, 1992; voir également Métaphysique contemporaine, E. Garcia et F. Nef (dir.), Paris, Vrin, 2007.

35. Meros peut s'entendre de plusieurs façons (Métaphysique, $\Delta, 25-26$ ): (i) un élément de la chose (pragma), (ii) un élément de sa définition (logos). Cf. Métaphysique, Z, 10, 1034 b 28. On retrouve donc ici la tension entre les deux interprétations des catégories, classes de choses ou de concepts.

36. Aristote, Catégories, R. Bodéüs (éd. et trad.), notes complémentaires, note 13, p. 80. 
Aristote, quoi qu'il en soit, introduit, à propos du critère d'inhérence, une relation ontologique authentique, celle de dépendance. On retrouvera plus loin cette relation à une place centrale dans la reconstruction que je proposerai. Cette relation sera posée dans le vocabulaire que je retiendrai entre particulier abstrait et particulier concret ${ }^{37}$. Ma position est d'affirmer qu'Aristote lie à tort la non-séparation des non-substances et l'absence de relation d'exemplification ou d'instanciation des universaux. Plus exactement il lie à tort ces relations à la séparation des universaux. L'étrangeté de l'objet de l'ontologie, relevée quasiment par tous les commentateurs, proviendrait en partie de cette tension.

\section{La carréification des catégories (Boèce, Averroès)}

Un carré, qu'il soit logique, sémantique ou ontologique se trouve au croisement d'une combinatoire et d'une ontologie relationnelle. En ce qui concerne ce dernier point la structure d'un carré est la suivante (avec trois types de relations: contradiction, contrariété, implication ou différence, négation et conséquence):

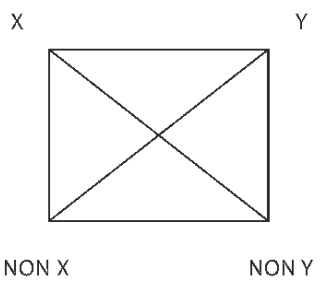

Par exemple «homme» et «femme» sont des contraires, «homme» et «non-homme» sont des contradictoires et «homme» implique «non-femme»:

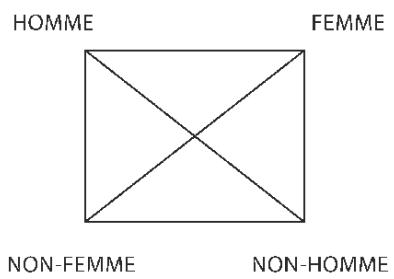

37. Pour une formalisation de la notion de dépendance, cf. Parts and Moments, B. Smith (éd.), Munich, Philosophia Verlag, 1982; et aussi F. Correia, Existential dependence and cognate notions, Munich, Philosophia Verlag, 2005. 
Je fonderai une partie importante de l'analyse sur deux distinctions, l'une déjà introduite un peu plus haut entre deux types d'ontologie, sur laquelle j'apporterai quelques précisions et une distinction que je voudrais introduire ici entre structure ontologique et structure sémantique. Une ontologie de constituant affirme que les items, comme les substances, les accidents, dont les particuliers tirent leurs caractères sont des composants ou des constituants des choses sensibles ${ }^{38}$, tandis qu'une ontologie relationnelle est une ontologie qui déduit des types de relations, participation, exemplification..., dans lesquelles entrent des particuliers, certaines des mêmes caractéristiques ${ }^{39}$.

Les structures ontologiques sont des complexes formés d'entités et de relations ${ }^{40}$. Par exemple, une structure ontologique peut réunir un ensemble de tropes (ou accidents individuels) et une relation de comprésence. La structure ontologique d'un particulier concret (comme un objet ou une personne) sera alors un ensemble de tropes unis par cette relation. La distinction entre structures ontologiques et structures sémantiques est parallèle à celle entre vérifacteurs et porteurs de vérité. Les structures ontologiques sont des vérifacteurs complexes. L'analyse du processus qui a conduit de la quadripartition des Catégories à la carréification (et, comme on le verra plus loin, à certaines approches catégorielles récentes) devra répondre aux deux questions suivantes, que notre mise au point conceptuelle, permet de rendre plus précises: les différentes formes de carré ontologique et de ramification catégorielle sont-elles des structures ontologiques ou des structures représentationnelles, c'est-à-dire seulement sémantiques?

Le point de départ de la carréification chez Porphyre et Boèce est la quadripartition des quatre classes d'étants dans Catégories, 2. Alain de Libera est parfaitement clair là-dessus:

La classification qui permet par combinaison de classer les quatre sortes d'étants: [essences] universelles, non [essences] particulières, non [essences] universelles, [essences] particulières - ce qui caractérise toute [essence] étant de n'être pas dans un sujet, toute non [essence] d'être dans un sujet, tout universel d'être dit d'un sujet, tout particulier de n'être pas dit d'un sujet.

38. Cf. M. Loux, «Aristotle's Constituent Ontology», p. 208.

39. Cette distinction a été introduite par l'ontologie de Gustav Bergmann chez qui se trouve implicitement cette distinction. Sur le nexus bergmanien, cf. F. Nef, "Connexion et relation chez Bergmann », in Gustav Bergmann. Phenomenological Realism and Dialectical Ontology, B. Langlet et J.-M. Monnoyer (éd.), Francfort, Ontos Verlag, 2009.

40. Cf. F. Nef, Traité d'ontologie pour les non-philosophes (et les philosophes), Paris, Gallimard (Folio essais; 525), 2009, chap. 7, p. 236 sq. 
Cette théorie des «quatre combinaisons» a été précisée par Porphyre, puis chez les Latins par Boèce (sous le nom de quatuor complexiones) ${ }^{41}$.

On peut remarquer que A. de Libera utilise prudemment les couples essences $v s$ non-essences et universel $v s$ particulier. Je ne suivrai pas la traduction de ousia par "essence» : " essence première» pour Socrate serait vraiment étrange. De plus, si je comprends ce qu'est une «non-substance», je ne comprends pas ce que c'est qu'une "non-essence»: un accident n'est pas une non-essence. La traduction de ousia par «essence " correspond à un souci qui n'est pas le mien ici.

Dans la littérature récente, trois présentations du carré ontologique coexistent, dans l'ordre d'apparition celles contemporaines de I. Angelelli ${ }^{42}$, de J. Vuillemin ${ }^{43}$ et celle de A. de Libera. I. Angelelli propose une mise en carré assez peu claire, mais qui a l'avantage de prendre comme base d'engendrement les relations «être dit de » (noté KU) et «être dans » (noté EU) :

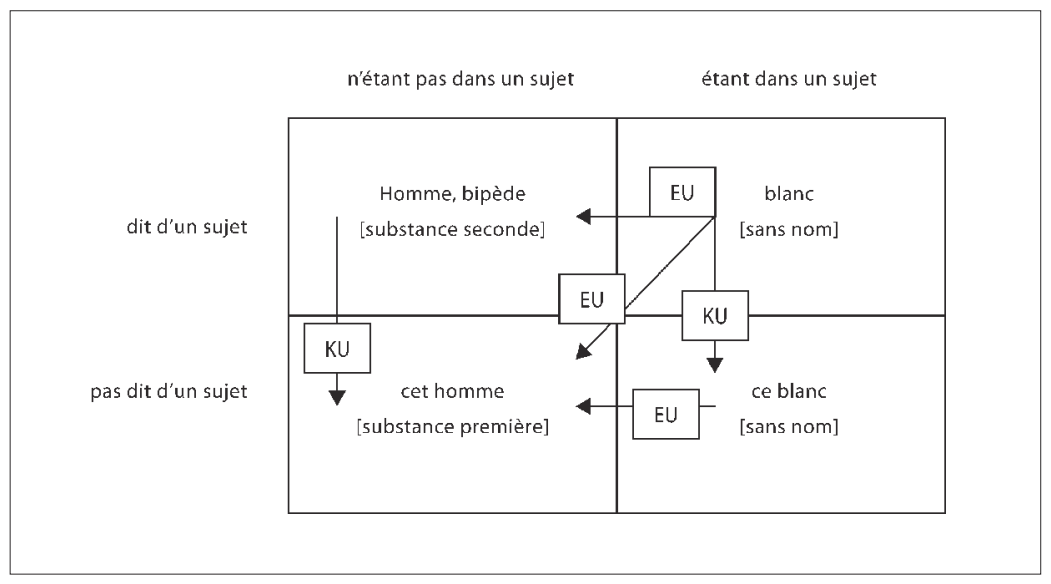

Le carré ontologique d'après Angelelli

41. A. de Libera, art. «Prédication», in Vocabulaire européen des philosophies, B. Cassin (dir.), Paris, Seuil, 2004.

42. I. Angelelli, Études sur Frege et la philosophie traditionnelle, J.-F. Courtine et al. (trad.), Paris, Vrin, 2007, p. 29.

43. J. Vuillemin, De la logique à la théologie: cinq études sur Aristote, Paris, Flammarion, 1967, p. 48. 
«Blanc» correspond à « universel» et «ce blanc» à accident particulier, dans le vocabulaire d'Angelelli, qui doute que cette dernière classe d'étant soit réellement l'inverse de «blanc». C'est la raison pour laquelle la portion de flèche qui va de «blanc» à «ce blanc» est barrée. Angelelli déclare:

[...] de simples raisons de symétrie inciteraient à admettre aussi des accidents particuliers comme relata de KU. [...] c'est là toucher d'emblée un point critique dans l'ontologie aristotélicienne et occidentale ${ }^{44}$.

Jules Vuillemin donne une autre présentation:

\begin{tabular}{|c|c|c|}
\hline Dicitur / Non & Universel & Singulier \\
\hline Abstrait & Généralités abstraites & $\begin{array}{c}\text { Particularités } \\
\text { abstraites }\end{array}$ \\
\hline Concret & Espèces, genres & Individus \\
\hline
\end{tabular}

(dicitur vs non dicitur est l'équivalent de KU, inest vs non inest de EU).

A. de Libera simplifie le tableau de J. Vuillemin:

\begin{tabular}{|c|c|c|}
\hline Dit d'un sujet & Dans un sujet & \\
\hline+ & + & Accident universel \\
\hline- & + & Accident particulier \\
\hline+ & - & Essence universelle \\
\hline- & - & Essence particulière \\
\hline
\end{tabular}

44. I. Angelelli, Études sur Frege..., p. 33. Pourquoi cette précision d'Angelelli? Est-ce que dans l'ontologie orientale les accidents individuels auraient une véritable place? La réponse à cette question est importante, mais déborde le cadre de cet article: il faudrait préciser ce qu'on entend par «oriental» (les Arabes sont-ils philosophiquement des orientaux? - les Perses sûrement, mais les Arabes d'Andalousie?) et même discuter de la pertinence de l'application du terme ontologie dans la pensée chinoise et indienne par exemple. De plus ces philosophies orientales recouvrent des gammes très étendues d'écoles avec des positions extrêmement contradictoires, du réalisme métaphysique le plus extrême au nominalisme radical, de l'idéalisme absolu au matérialisme complet et la réponse à la question d'Angelelli serait certainement complexe.

45. Variante lexicale des «particuliers abstraits»? 
Ce tableau est en fait un aide-mémoire combinatoire du carré ontologique de Boèce ${ }^{46}$ :

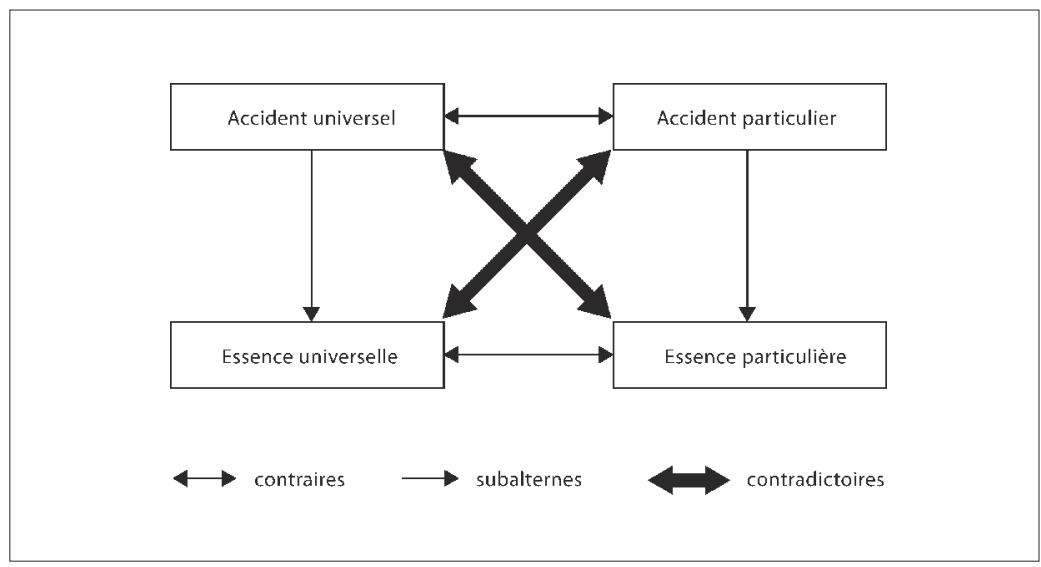

La concordance entre les reconstructions de J. Vuillemin et de A. de Libera est la suivante:

- généralité abstraite = accident universel,

- particularité abstraite $=$ accident particulier,

- espèce, genre, différence = essence universelle,

- individu $=$ essence particulière.

La classification de J. Vuillemin présente l'avantage d'insister, à juste titre selon moi, sur l'opposition abstrait $v s$ concret et celle de A. de Libera le défaut de proposer ou reprendre une opposition problématique entre essence universelle et particulière. Il y a des essences générales et individuelles, des universaux et des particuliers, mais il est beaucoup moins clair de savoir ce qu'est une essence universelle ou une essence particulière, même si ces termes se trouvent chez tel ou tel commentateur. Je préfère privilégier une reconstruction qui éclaire le débat contemporain, plutôt que de me risquer à une hypothétique archéologie. Je proposerai donc le carré suivant, en tenant compte de l'ordre d'exposition du texte d'Aristote, et en tenant compte du fait que le critère d'indépendance sert à distinguer le concret et l'abstrait ${ }^{47}$ («D» $=$ dit de; «I» = est dans):

46. On laisse de côté le fait, bien documenté dans le commentaire de C. Luna au commentaire de Simplicius (Simplicius, Commentaire sur les Catégories...) que Boèce apparemment a fait un contresens célèbre sur la division proposée par Porphyre.

47. Cf. I. Angelelli, Études sur Frege..., p. 29. 


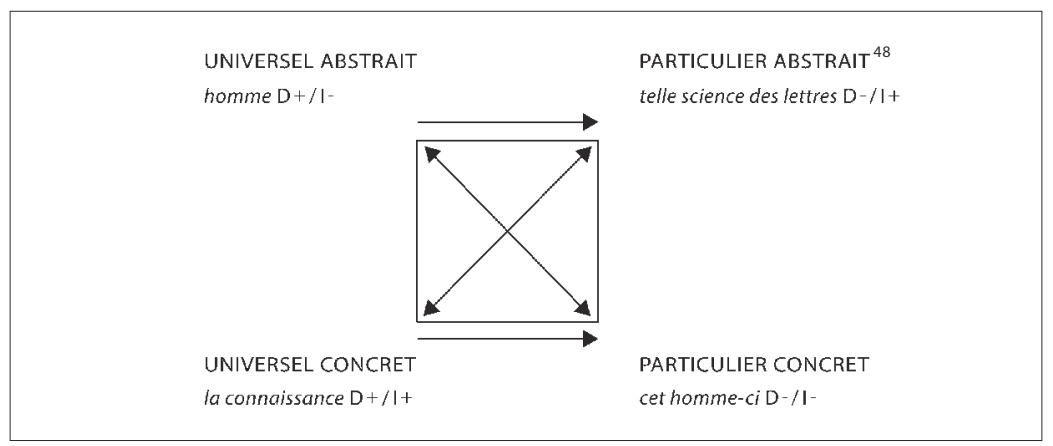

Le carré ontologique de Boèce a une longue histoire (retracée par les historiens des doctrines médiévales, comme A. de Libera). Il est marqué par un défaut grave, comme le souligne I. Angelelli:

Malheureusement Boèce $[\ldots]$ ne fournit pas d'explication sur la manière de combiner les deux divisions et les deux relations fondamentales (singulier - universel, substance - accident) ${ }^{49}$.

Averroès cherche à pallier ce défaut en proposant le carré ontologique suivant:

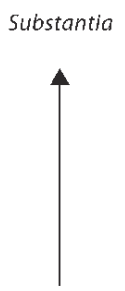

Universale

\section{Inconsistens}

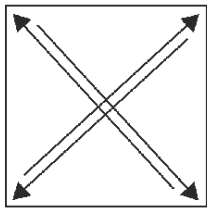

Inconsistens
Accidens

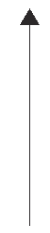

Particulare
De substantia dicitur et in substantia non est
De substantia non dicitur et in substantia est

48. Dans «Platonisme et particularisme...», p. 159-186, j'ai soutenu qu' il n'y avait pas de particulier abstrait ou de trope au sens strict chez Aristote, ce que je serai toujours prêt à soutenir. Mais ici le point de vue est différent. Je souhaite reconstruire la doctrine des catégories, pour disposer d'un espace conceptuel de comparaison des doctrines anciennes et modernes. Disons que Catégories, 2, comprend une place pour les particuliers abstraits, mais que la métaphysique aristotélicienne dans son ensemble n'a pas exploité systématiquement cette possibilité.

49. I. Angelelli, Études sur Frege..., p. 29, n. 1. 
Le particulier n'est pas dit de la substance et n'est pas dans la substance (nec de substantia dicitur nec in substantia est) et l'accident est dit de la substance et est dans la substance (de susbstantia dicitur et in substantia est). La relation substance/ universel et celle entre accident et particulier sont de consistance ${ }^{50}$.

La carréification, très brièvement évoquée ici, pose beaucoup de problèmes. Tout d'abord le carré ontologique a probablement sa source dans le carré logique (qui sert par ailleurs également de patron pour les modalités du possible et du nécessaire):

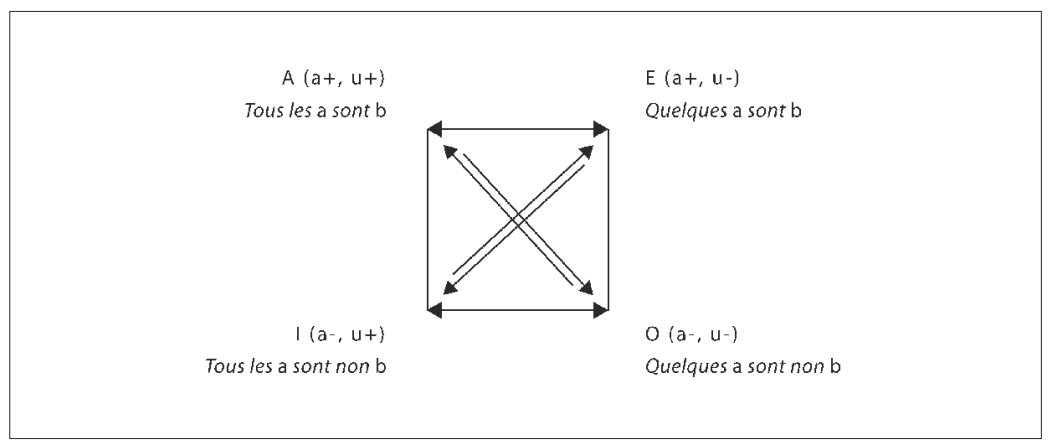

A et $\mathrm{E}, \mathrm{I}$ et $\mathrm{O}$ sont des contradictoires; $\mathrm{A}$ et $\mathrm{O}$, I et $\mathrm{E}$ des contraires.

Or, les relations de contrariété et de contradiction sont des relations entre propositions, pouvant ou ne pouvant pas être fausses ou vraies en même temps, alors que les relations entre les étants selon l'interprétation même des Catégories ne peuvent pas être des relations logiques. Un accident ou un abstrait n'est ni vrai, ni faux, les relations ontologiques (ou les relations candidates à ce titre) comme l'instanciation, l'exemplification, la dépendance ne sont pas des relations logiques de contradiction ou de contrariété. On voit dans le carré ontologique d'Averroès ci-dessus une tentative de traduire la contradiction et la contrariété logiques en inconsistance ontologique.

Jean-Baptiste Rauzy ${ }^{51}$ en reprenant les deux dimensions de l'ontologie dégagées par Aristote propose le schéma suivant:

50. Ce schéma est cité dans J. Vuillemin, De la logique à la théologie..., p. 48. Il est tiré de l'édition de Venise de 1562 d'Averroès (Aristote, Aristotelis opera cum Averrois commentariis, rééd., Francfort, Minerva, 1962, vol. 1, p. 24).

51. J.-B. Rauzy, La doctrine leibnizienne de la vérité, Paris, Vrin, 2001, p. 244. J'ai critiqué, d'un autre point de vue l'utilisation de ce schéma pour l'ontologie de Leibniz dans F. Nef et D. Berlioz, Leibniz et les puissances du langage, Paris, Vrin, 2005, p. 128 sq. 


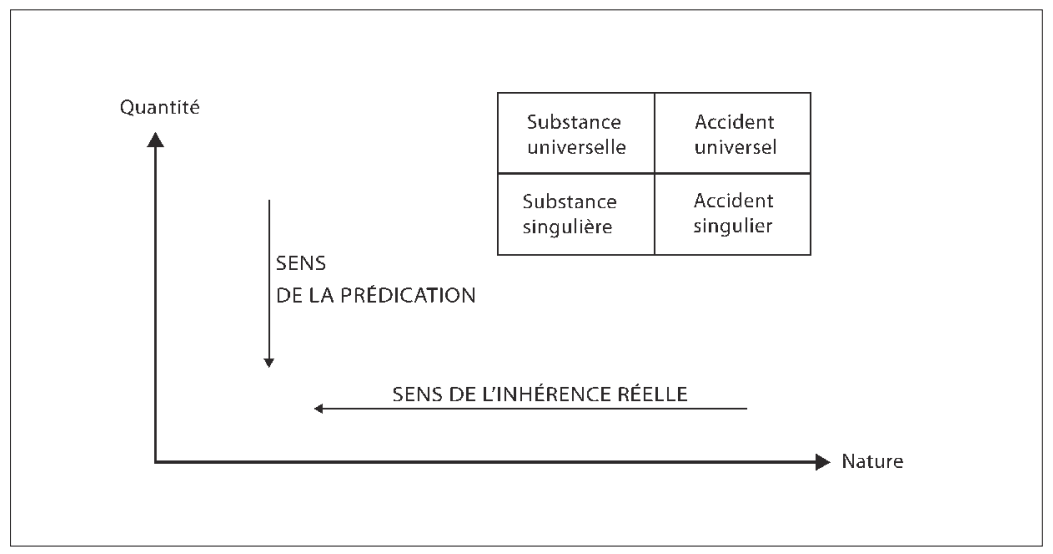

J.-B. Rauzy distingue ici une première relation d'ordre qui est la hiérarchie des genres et des espèces et une relation horizontale qui est celle de l'inhérence réelle qui part toujours d'un accident:

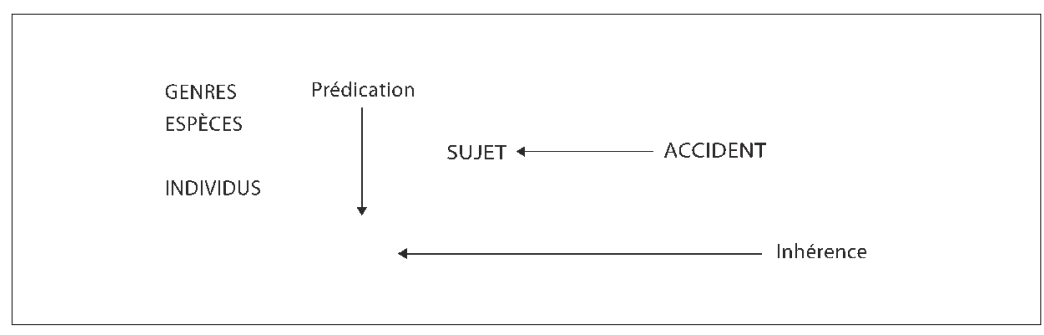

On peut voir les différentes tentatives de carréifier les distinctions des Catégories comme autant de recherches en vue de dégager une structure ontologique, sur le modèle du carré logique. Cependant, il est douteux que le carré ontologique constitue à soi seul une telle structure ontologique. Si en effet on part de la quadripartition telle qu'on l'a décrite et analysée, on obtient par décomposition, i. e. abstraction sur la structure, les relations:

(1) le particulier abstrait dépend du particulier concret,

(2) l'universel abstrait est exemplifié dans le concret particulier,

(3) l'universel abstrait est instancié dans le particulier abstrait.

La différence entre instancier et exemplifier est la suivante:

- l'exemplification est la relation d'un objet universel abstrait à un concret particulier. Définition : $x$ exemplifie y si y est un universel et $\mathrm{x}$ un 
particulier concret qui possède la propriété y. Exemple: ce cahier qui est rouge exemplifie le rouge;

- l'instanciation est une relation entre un universel et un particulier abstraits: le rouge est instancié dans ce rouge, ou ce rouge particulier est une instance de rouge. Définition : $\mathrm{x}$ instancie y si $\mathrm{x}$ est un particulier abstrait, qui est une partie de l'universel. Exemple: le rouge de ce cahier est une instance du rouge (ou: une instance de rouge).

J'ai proposé ailleurs ${ }^{52}$ le carré ontologique suivant en prenant un prédicable unique: «rouge» et en essayant de donner un sens à la distinction entre universel abstrait et concret, parallèle à celle entre particulier concret et abstrait, par exemple quand elle est lexicalisée comme pour rouge $v s$ rougeur, distinction responsable du contraste de certaines acceptabilités sémantiques:

- la rougeur de son visage m’inquiète,

- * le rouge de son visage m'inquiète,

- le rouge m'est monté aux joues,

- * la rougeur m'est montée aux joues.

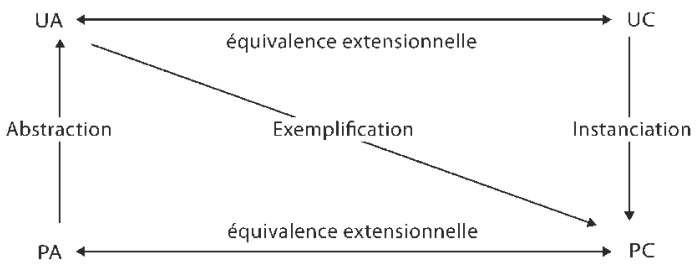

Dans ce carré $\mathrm{UA}=$ universel abstrait (ex. : le rouge); $\mathrm{UC}=$ universel concret (ex. : la rougeur); PA = particulier abstrait (ex. : tel rouge, c'està-dire le rouge de $\mathrm{x}$ pris abstraitement par rapport à $\mathrm{x}) ; \mathrm{PC}=$ particulier concret (ex. : le rouge-de-x).

C'est peut-être le moment de faire deux remarques triviales. La première est qu'à une carréification correspond formellement une ramification, sans que le contraire soit forcément vrai. Par exemple, à la carréification ci-dessus correspondent les deux ramifications:

52. F. Nef, Les propriétés des choses..., p. 220-222. 


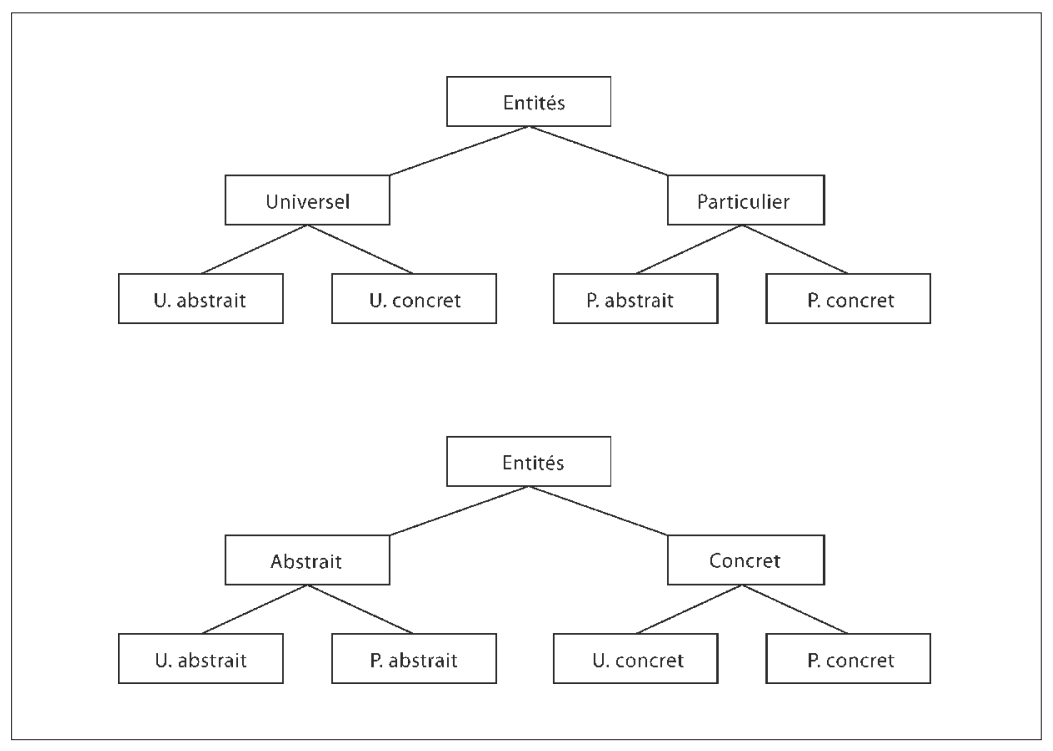

Ces deux ramifications sont équivalentes; elles ne diffèrent que par le choix de la division primitive (abstrait $v s$ concret ou universel $v s$ particulier).

E. J. Lowe ${ }^{53}$ montre également comment deux distinctions engendrent quatre catégories ( "how two distinctions generate four categories»). Il dégage implicitement une structure ontologique, puisqu'il adjoint aux éléments (substances et propriétés individuelles et universelles) des relations ontologiques (caractérisation, instanciation). La structure ontologique qu'il propose est donc la suivante:

$$
(<\mathrm{S}>,<\mathrm{P}>, \mathrm{C}, \mathrm{I})
$$

où $<\mathrm{S}>$ est un ensemble de substances, $<\mathrm{P}>$ un ensemble de propriétés et $\mathrm{C}$ et I les deux relations que nous venons de mentionner et qui sont selon lui des relations formelles, transcendantales ${ }^{54}$. Le problème toutefois avec cette interprétation du carré me semble être le suivant. L'universel et le particulier ne peuvent apparaître dans la structure ontologique au rang des propriétés (ce sont des propriétés tout à fait différentes des propriétés et relations qui servent à structurer les substances, ce sont des propriétés formelles générales). Or, si les propriétés caractérisent les substances (relation $\mathrm{C}$ entre $<\mathrm{S}>$ et $<\mathrm{P}>$ ) qu'en est-il de I? E. J. Lowe refuse qu'il y ait des universaux dans sa substance. Les propriétés sont des manières d'être

53. E. J. Lowe, The Four-Category Ontology, p. 57 sq.

54. Ibid., p. 59-60. 
des objets et les propriétés formelles générales sont finalement aussi des manières d'être: l'universel et le particulier sont eux aussi des ways of being. La conséquence est qu'il faudra pour bâtir le carré ontologique poser des universaux aux coins supérieurs de la figure, alors que l'ontologie de Lowe devrait probablement refuser de le faire: il y a une tension entre l'intention de l'ontologie et sa forme, exactement comme chez Aristote. On peut remarquer, comme on l'a signalé rapidement plus haut que la converse n'est pas vraie: toute ramification n'est pas carréificable. Par exemple, à la ramification suivante ne correspond pas un carré ontologique:

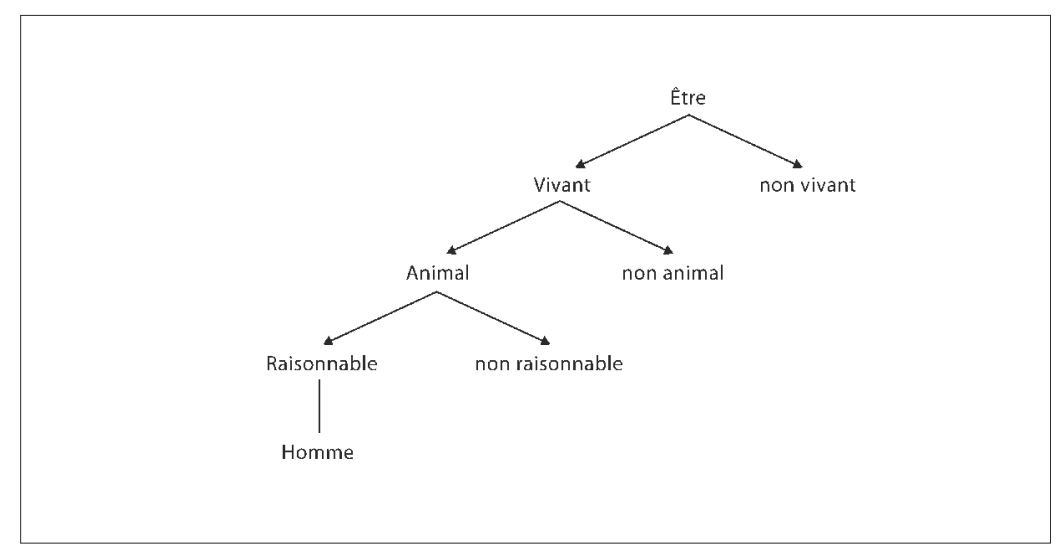

La raison est simple: cet arbre de Porphyre a une profondeur $>2$. Un carré ne peut correspondre qu'à un arbre d'une profondeur $=2$, comme l'un des deux arbres ci-dessus avec UA, PA, UC, PC.

Avec une profondeur $=3$ on a un cube logique $\left(2^{3}=8\right)$ :

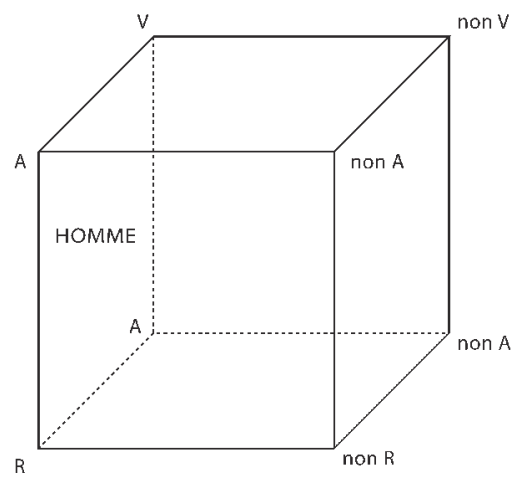


On est obligé de répéter une ligne (car $2 \times 3=6$ et il y a 8 sommets). Cela n'est pas grave car la face qui a pour sommets $\mathrm{V}$, A et $\mathrm{R}$ a une redondance du sommet A, ce qui n'a pas d'importance. On peut remarquer que la face opposée serait l'espace de la chose. Par contre, les faces supérieure et inférieure ne correspondent pas à des espaces conceptuels ou ontologiques, car les sommets sont des entrées contradictoires. Peut-être pour toutes ces raisons les cubes logiques sont-ils une rareté dans la littérature. On peut aussi s'expliquer la perfection du carré logique $: 2^{2}$ est le nombre des places sur le carré et 2 est le nombre de lignes, ce qui implique qu'on ne doive pas répéter de ligne. La fascination pour le carré proviendrait donc peut-être de propriétés mathématiques triviales.

En effet un arbre qui correspond à un carré sans reste, ni répétition a la forme suivante:

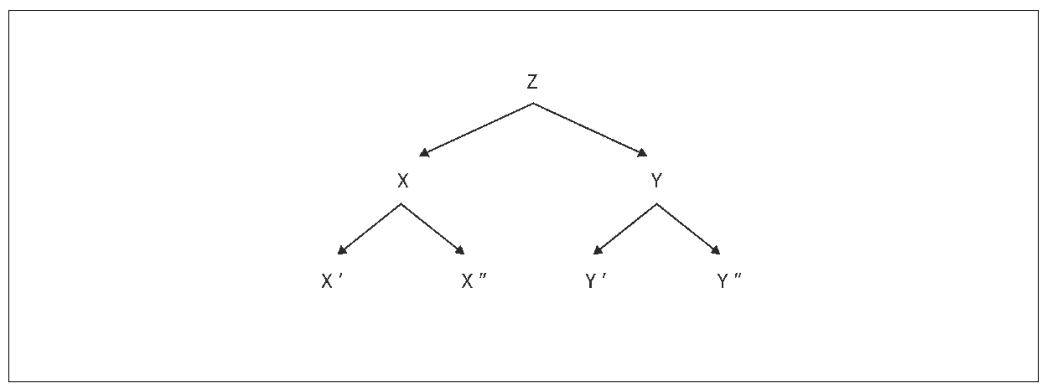

où le nombre des lignes est égal au nombre des nœuds, ce qui est parfait et rend possible la carréification:

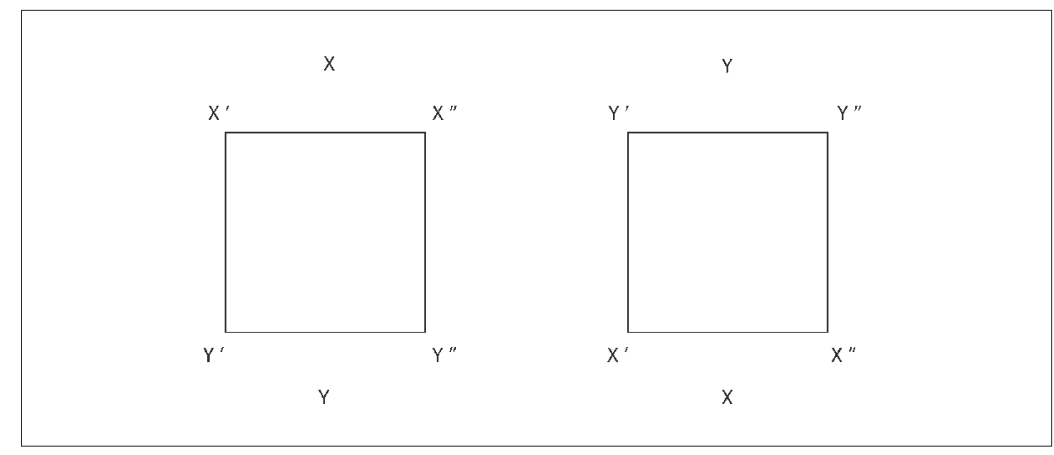

Une dernière remarque: le fait qu'à un arbre (à une divisio entis) corresponde deux carréifications équivalentes explique qu'au Moyen Âge on ait débattu de la différence entre la prima divisio (entre substantia et accidens) 
et la secunda divisio (entre universale et particulare). On peut interpréter la prééminence de la prima divisio entis sur la deuxième, par une supériorité de la division ontologique sur la division logique qui a un aspect ontologique, ne se réduit pas à une simple division conceptuelle.

\section{Carréification $v s$ ramification : l'ontologie quadri-catégorielle de E. J. Lowe}

E. J. Lowe défend une orientation aristotélicienne et donc une ontologie à quatre catégories fondamentales qui accepte la primauté catégorielle de la substance individuelle. Il introduit par rapport aux carrés que nous avons examinés deux innovations: la première est importante; elle consiste à introduire des sortes (kinds) conçues comme des universaux substantiels (à peu près l'équivalent d'universaux concrets?). La seconde, terminologique, consiste à appeler «modes» les particuliers abstraits (appelés aussi «tropes»):

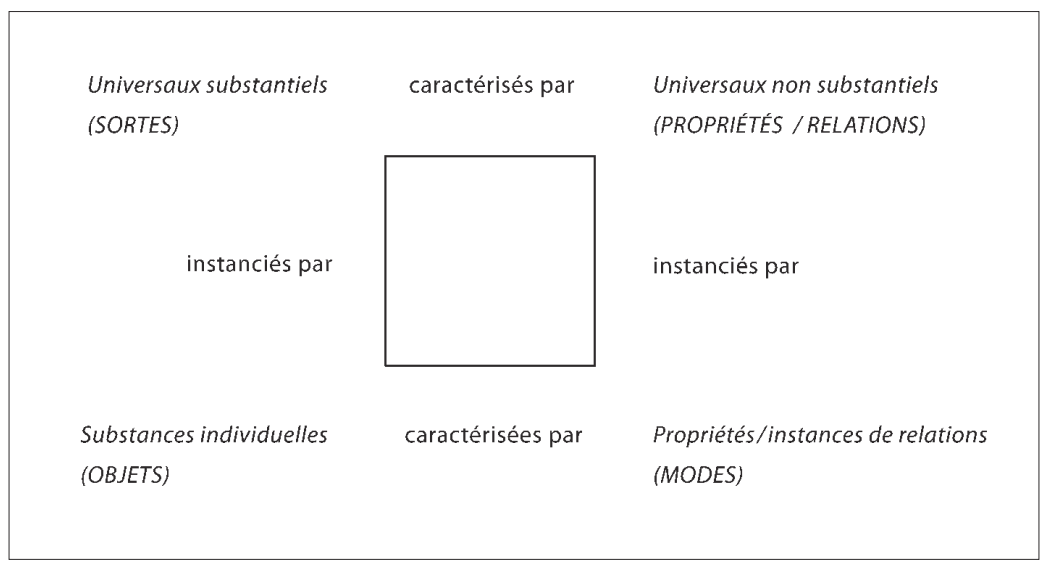

Le carré ontologique de Lowe

On peut remarquer que E. J. Lowe nomme «modes» (modes) les instances de propriétés et de relations, ou en d'autres termes des propriétés et des relations instanciées, parce qu'il refuse de faire des tropes des constituants de particuliers, comme le font les particularistes: selon lui les propriétés générales ou instanciées sont des manières (ways) d'être. J’ai critiqué plusieurs fois cette manière de voir car cette caractérisation des propriétés me semble s'appliquer seulement à un sous-ensemble de propriétés. Il me semble curieux, en effet de soutenir que la masse d'un corps est une manière d'être de ce corps, alors qu'être rouge pour une pomme peut évidemment 
être conçu de manière plus intuitive ou évidente comme une manière d'être de la pomme en question. On peut peut-être établir une correspondance entre la masse et la propriété d'avoir un poids, «être pesant» pouvant être considéré comme une manière d'être, mais il me semble qu'on a intérêt à bien distinguer les deux choses, la propriété naturelle d'avoir une masse et la qualité d'être pesant.

On peut remarquer que ce carré ontologique de E. J. Lowe peut être ramifié de deux façons qui correspondent à la prima et à la secunda divisio entis:

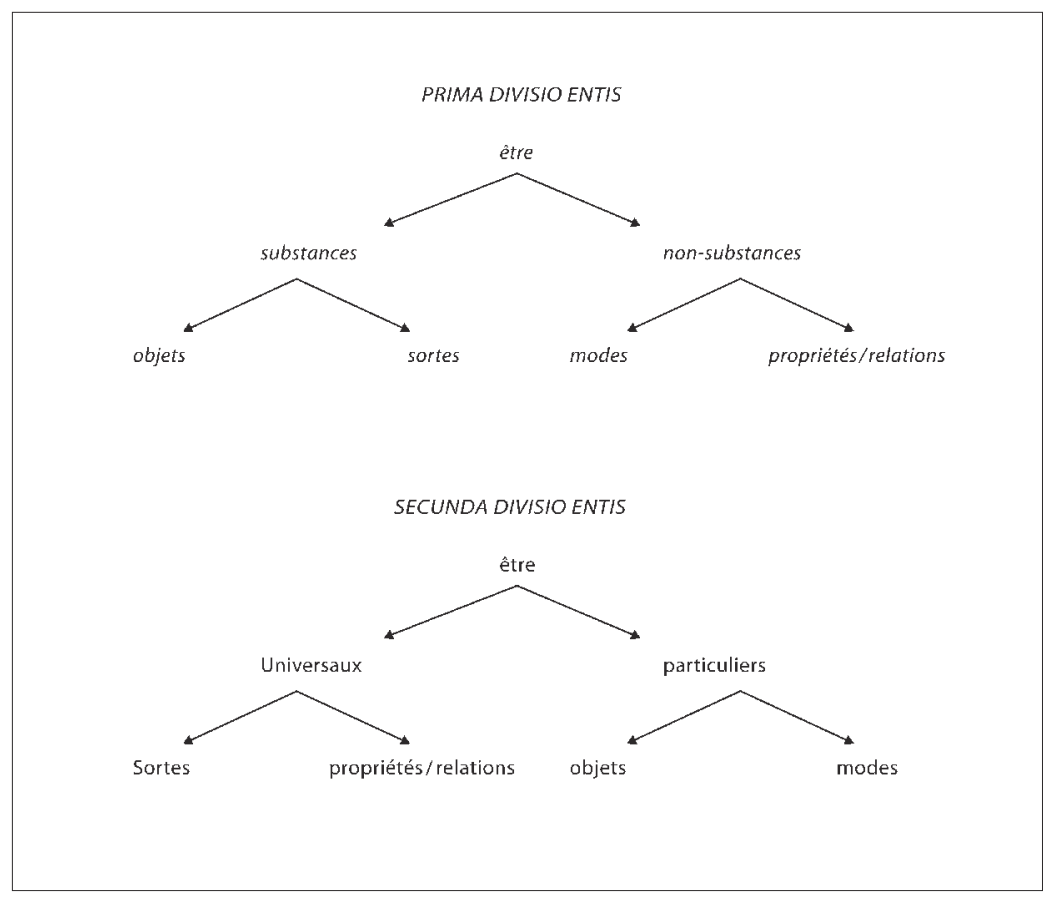

Le carré ontologique de E. J. Lowe combine les deux divisions: par exemple, un objet est un particulier (prima divisio) et une substance (secunda divisio). On peut noter à propos de l'objet que ce dernier étant un particulier, il ne saurait y avoir des objets abstraits, et qu'étant une substance, il ne saurait y avoir d'objet non substantiel, ce qui est gênant d'une part pour les mathématiques ${ }^{55}$ et d'autre part pour la physique quantique. On voit

55. Cela dit, dans «Essences» (R. Pouivet (trad.), in Métaphysique Contemporaine, p. 85-117), Lowe défend un type de fictionalisme pour les ensembles et les nombres. 
mal où placer un nombre imaginaire ou une particule élémentaire, alors même que cette ontologie catégorielle est proposée pour servir d'ontologie de la science. C'est tout le problème du réalisme scientifique qui est posé là, problème qu'on laisse de côté 56 .

E. J. Lowe avant son carré ontologique a proposé une ramification - dans notre vocabulaire il est passé d'une représentation porphyrienne en 1998 à une représentation boécienne en 2006 :

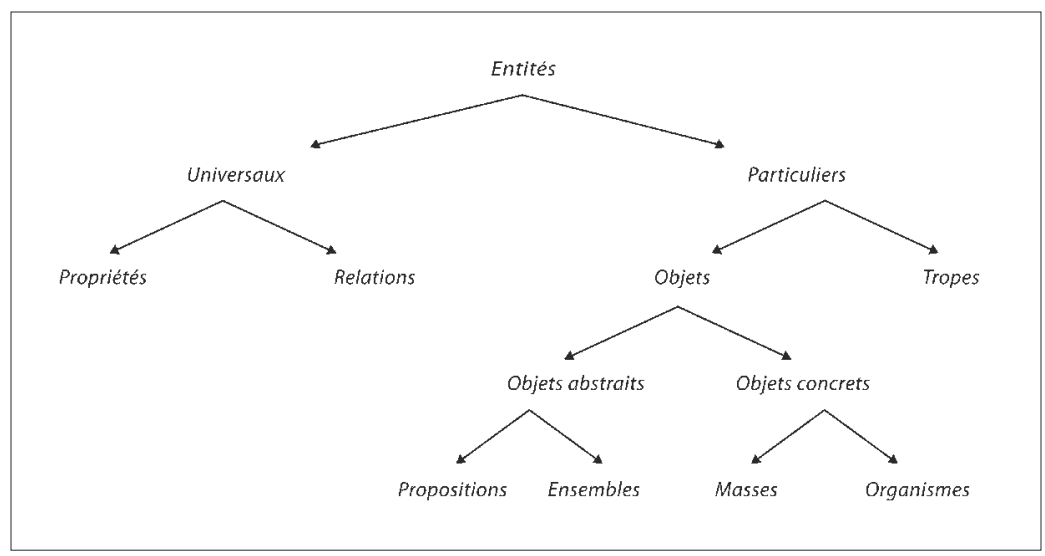

Un fragment de la hiérarchie des catégories

Cette ramification détaille les objets qui sont les éléments de base de l'ontologie. On remarque que le couple abstrait $v s$ concret s'applique (seulement) aux objets et pas aux universaux et particuliers, à l'inverse de la division que j'ai proposée plus haut. Les modes et les tropes ne sont pas en effet dans ce tableau identiques strictement à des particuliers abstraits. La division de l'abstrait et du concret est donc hiérarchiquement inférieure à celle du particulier et de l'universel, à celle de l'individu et de la substance. La division de l'abstrait et du concret est ajoutée à la combinaison de la divisio prima et de la divisio secunda.

56. Il existe cependant une version faible du réalisme scientifique qui est probablement compatible avec ce type d'ontologie; elle consiste à poser comme norme de l'explication métaphysique que celle-ci ne doit pas contredire explicitement les investissements ontologiques de la science. Par exemple, une métaphysique qui nierait l'existence des événements se placerait probablement dans ce cas en niant l'investissement ontologique de la théorie de la relativité restreinte. Au sens fort, le réalisme scientifique signifie que l'ontologie est une explicitation de la science et que par exemple les structures physiques sont par elles-mêmes des structures ontologiques. 
On peut comparer cette division avec celle que propose R. Chisholm ${ }^{57}$ :

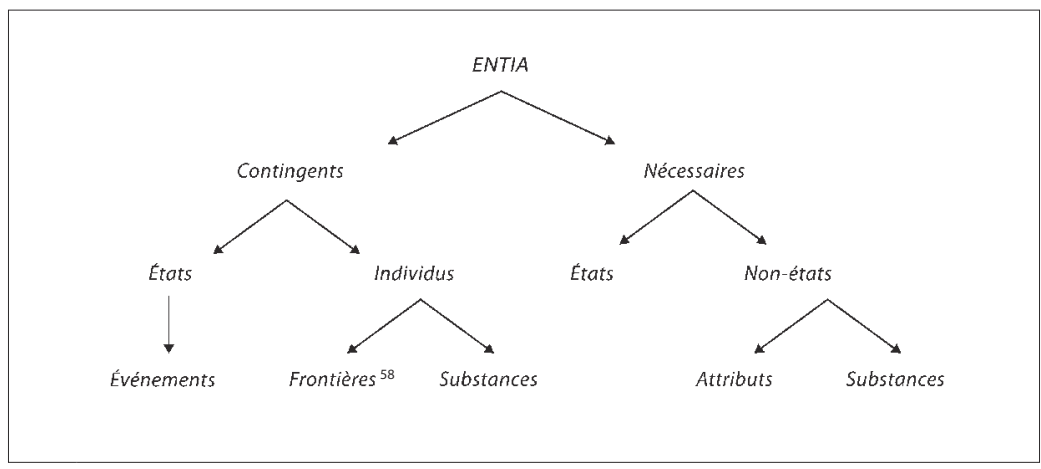

La première division est une division modale (entre contingent et nécessaire). La raison en est que techniquement " $\mathrm{x}$ est nécessairement tel qu'il est $\mathrm{F}$ » ( $x$ is necessarily such that it is $F$ ) est un primitif de la théorie, i. e. est non défini ${ }^{59}$. Mais derrière cette raison technique il y a une raison plus profonde: l'ontologie chisholmienne est intentionnelle et donc intensionnelle. Les attributs qui avec les événements et les substances forment la base ontologique de la théorie ont une structure intentionnelle ${ }^{60} . \mathrm{La}$ division de l'être en universel et particulier chez Lowe est extensionnelle (ou peut-être neutre suivant la conception que l'on a des universaux), tandis que la division de l'être entre nécessaire et contingent chez Chisholm est intensionnelle.

\section{Conclusion}

En un certain sens on peut affirmer que les catégories sont bien des diviseurs de l'être. Plus précisément les catégories aristotéliciennes correspondent bien à un classement des étants et ces catégories elles-mêmes dépendent de structures ontologiques où figurent des universaux et des particuliers, des abstraits et des concrets, avec des relations d'instanciation et d'exemplification. Les

57. R. Chisholm, A Realistic Theory of Categories : an Essay in Ontology, Cambridge, Cambridge University Press, 1996, p. 3.

58. Cette catégorie recouvre les frontières et les surfaces (ces dernières sont des frontières à deux dimensions). L'origine brentanienne de ce concept est claire: Brentano a développé une théorie de la plérose (plerosis) à propos des frontières et du continu. Cf. F. Brentano, "Das Kontinuum», in Philosophische Untersuchungen zu Raum, Zeit und Kontinuum, Hambourg, Felix Meiner Verlag, 1976, p. 3-59.

59. R. Chisholm, A Realistic Theory of categories..., p. 5-7.

60. Ibid., p. 29-35. 
catégories aristotéliciennes dans la maxima divisio ${ }^{61}$ peuvent être entendues au sens de formes générales de questions: où, quand? comment? etc. ${ }^{62}$, mais dans le chapitre des Catégories que nous venons d'examiner, consacré à la minima divisio, les catégories obéissent au classement fondamental de la substance et de la non-substance, de l'universel et du particulier. Aristote s'efforce d'obtenir les catégories à partir d'une combinatoire restreinte de relations «être dans " et "être dit de", une relation tout / partie et une relation de prédication. Par exemple, la substance n'est ni dans autre chose, ni dite d'autre chose. En prenant des relations comme base de sa combinatoire Aristote a fondé l'ontologie, comme classement formel des étants, sur la distinction même de ces relations, sur une base sémantique et méréologique à la fois. C'est en ce sens que le carré ontologique est une étape importante de l'histoire de l'ontologie. Mais d'autres questions se posent au métaphysicien d'aujourd'hui. La première est sans doute celle de l'existence ou de l'inexistence d'entités à la fois non substantielles et individuelles (Callias's generosity, un exemple de propriété ackrillienne). La seconde question qui se pose est la nature intensionnelle ou extensionnelle des catégories: sont-elles dépendantes de l'esprit? Cette question est différente de celle qui touche à leur statut conceptuel ou ontologique: on peut imaginer des catégories qui seraient des concepts, mais non dépendantes de l'esprit, des catégories platoniciennes, éternelles et innées; on peut imaginer à l'inverse tout aussi bien des catégories à portée ontologique dépendantes de l'esprit, si ces catégories étaient conçues comme des projections anthropomorphes des facultés psychologiques découpant le monde aux bonnes jointures.

Frédéric NeF

EHESS \& Institut Jean-Nicod

61. Les commentateurs de l'Antiquité distinguent deux divisions: une division maximale, celle en dix catégories et une division minimale, en quatre classes d'êtres. Cf. Simplicius, Commentaire sur les Catégories..., p. 140 sq., pour l'origine chez Porphyre de cette distinction.

62. J. Hintikka et ses élèves ont analysé les catégories aristotéliciennes de ce point de vue à l'intérieur de la sémantique fondée sur la théorie des jeux (game theoretical semantics ou GTS). Cf. dans mon article sur les catégories brentaniennes, «La lecture par Brentano... », p. 3 sq., une présentation de l'approche de Hintikka. Cf. aussi J. Hintikka et J. Kulas, The Game of Language, Dordrecht, Reidel, 1983. 